\title{
A revealed reference point for prospect theory
}

\author{
Katarzyna M. Werner ${ }^{1}$ - Horst Zank ${ }^{2}$ (D)
}

Received: 14 November 2016 / Accepted: 19 December 2017 / Published online: 13 January 2018 (C) The Author(s) 2018. This article is an open access publication

\begin{abstract}
Without an instrument to identify the reference point, prospect theory includes a degree of freedom that makes the model difficult to falsify. To address this issue, we propose a foundation for prospect theory that advances existing approaches with three innovations. First, the reference point is not known a priori; if preferences are reference-dependent, the reference point is revealed from behavior. Second, the key preference axiom is formulated as a consistency property for attitudes toward probabilities; it entails both a revealed preference test for reference-dependence and a tool suitable for empirical measurement. Third, minimal assumptions are imposed for outcomes, thereby extending the model to general settings. By incorporating these three features we deliver general foundations for prospect theory that show how reference points can be identified and how the model can be falsified.
\end{abstract}

Keywords Probability midpoint $\cdot$ Prospect theory $\cdot$ Rank-dependence $\cdot$ Reference point $\cdot$ Sign-dependence

JEL Classification D03 - D81

An earlier version was titled "Foundations for Prospect Theory Through Probability Midpoint Consistency." We are indebted to Mohammed Abdellaoui, Manel Baucels, Rani Spiegler and Peter Wakker for extensive comments on the earlier version. We also acknowledge the comments of anonymous referees that led to further improvements.

Horst Zank

horst.zank@manchester.ac.uk

1 Manchester Metropolitan University, Manchester, UK

2 Department of Economics, University of Manchester, Oxford Road, Manchester M13 9PL, UK 


\section{Introduction}

Prospect theory (PT; Tversky and Kahneman 1992) is regarded as one of the most successful descriptive theories for risk and ambiguity (Starmer 2000; Kahneman and Tversky 2000; Wakker 2010; Barberis 2013). Relative to classical expected utility (EU), PT incorporates nonlinear treatment of probabilities (Preston and Baratta 1948; Allais 1953; Quiggin 1982), or nonadditive event uncertainty resulting from ambiguity (Ellsberg 1961; Schmeidler 1989) and reference-dependence. The latter requires the existence of a reference point, relative to which outcomes are seen as gains or losses, and constitutes a distinctive feature and a key assumption of PT. What exactly determines the reference point has been left unspecified, and not offering a plausible explanation for how the reference point is derived from primitives, i.e., from preferences over prospects, is regarded as a major shortcoming of PT (Fudenberg 2006, p. 696 footnote 2; Pesendorfer 2006, pp. 713-716).

We develop a revealed preference technique based on probability midpoints to show that PT can be obtained from preferences without assuming the reference point as exogenously given. Starting from an indifference between two prospects, probability midpoints are obtained by shifting probability mass across outcomes, such that a new indifference results (Kuilen and Wakker 2011). By keeping the outcomes of these prospects ordered in terms of preference and commonly fixed, and repeatedly shifting probability mass across adjacent outcomes, one can elicit a sequence of probabilities that are perceived equally far apart in terms of preferences. ${ }^{1}$ If the reference point is meaningful for preferences, the elicited probability midpoints will be affected when probability mass is shifted from losses to gains. This can be used to reveal the location of the reference point. Indeed, this feature of behavior is exploited to show how the reference point in PT is revealed from choices. It is in this sense that we obtain the reference point endogenous to the model.

To position our contribution, let us recall that the von Neumann and Morgenstern (1944) foundations for EU imply a linear treatment of probabilities. This means that elicited probability midpoints are arithmetic midpoints and independent of the type or magnitude of outcomes. The EU-axioms do not impose a specific interpretation for outcomes or restrictions on utility, except for the latter being a cardinal measure. For instance, one can interpret real-valued outcomes as final wealth positions (a frequent assumption made in theoretical applications) or as changes relative to a reference point (usually an implicit assumption made in experimental studies). ${ }^{2}$ In particular, imposing a utility value of 0 at the reference point, and treating outcomes with negative utility values as losses and those with positive utility as gains, is compatible with the

\footnotetext{
1 This elicitation method is similar to the (dual analog) elicitation technique for utility measurement, where standard sequences of equally spaced outcomes on the utility scale are obtained (Wakker and Deneffe 1996). For PT-preferences, probability midpoints are equally spaced on the corresponding probability weighting scales.

2 That the EU-axioms were not tied to a specific interpretation of outcomes was also noted by Kahneman and Tversky (1979, p. 264). A similar argument holds for the rank-dependent utility model of Abdellaoui (2002) who uses the von Neumann and Morgenstern framework as we do. Although Abdellaoui's preference conditions allow for a nonlinear treatment of probabilities, they do not restrict the cardinal family of utility functions in any way nor do they require a specific interpretation for outcomes.
} 
EU-axioms as long as probabilities are treated linearly. The requirement that utility is 0 at the reference point then appears as an arbitrary restriction of the class of admissible cardinal utilities to a smaller ratio-scale subclass. By contrast, in PT such restrictions on admissible utility functions follow from the asymmetric treatment of the probabilities attached to gains and losses and, thus, properties that capture deviations from linearity in probabilities. It is precisely this asymmetric nonlinear treatment of probabilities, revealed as an inconsistency in elicited probability midpoints, that we exploit in order to provide general foundations for PT.

A nonlinear treatment of probabilities has also been incorporated into the rankdependent utility (RDU) model (Quiggin 1981, 1982; Segal 1987; Wakker 1994). RDU can be seen as a special case of PT where the presence of a reference point is immaterial for attitudes toward probabilities. General foundations for RDU were provided by Nakamura (1995), Abdellaoui (2002), Abdellaoui and Wakker (2005), and Zank (2010), and they can readily be used to derive PT if the reference point is known in advance. Without knowing that a reference point exists, deriving PT from RDU becomes a challenge. To achieve foundation for PT, we employ a consistency test for specifically elicited probability midpoints. Consistency means that the treatment of probabilities is insensitive to replacements of the stimuli used to elicit midpoints. As our consistency property does not impose restrictions on the admissible probability weighting functions under PT, probability midpoint consistency can accommodate a wide range of behaviors (e.g., risk behavior captured through the popular inverse-Sshaped probability weighting functions; Prelec 1998).

To give further intuition for our main preference tool, suppose we have identified the reference point. Then our condition requires that probability midpoints elicited from preferences are independent of the outcomes (i.e., the stimuli used in the elicitations), whenever the latter are of the same type (i.e., either they are all gains or they are all losses). This is a natural requirement for the treatment of probabilities under PT, where a distinct nonlinear treatment for probabilities of gains as compared to probabilities of losses is explicitly allowed for; it can be inferred, for example, from the reflection examples in Kahneman and Tversky (1979, p. 268). This feature of reference-dependent behavior, which we call sign-dependence, has been widely documented. ${ }^{3}$ Sign-dependence can be inferred from the presence of distinct probability midpoints for gains than for losses, as implied by empirically elicited parametric forms (e.g., Tversky and Kahneman 1992; Abdellaoui 2000). Conversely, sign independence of probability midpoints suggests that the reference point is immaterial for the treatment of probabilities (as in EU or RDU). Hence, if we do not know the location of the reference point, we can employ probability midpoints elicited for different outcomes to test for sign independence. This leads to a revealed preference technique, where replacing a gain by a different gain does not affect elicited probability midpoints and

\footnotetext{
3 For monetary outcomes, the term "sign-dependence" is sometimes used to indicate that the utility for gains (i.e., positive outcomes) reveals a different shape than the utility for losses (negative outcomes), e.g., concave versus convex. Here we use the term "sign-dependence" to indicate that the weighting functions under PT generate different weights for negative utility (from losses) as compared to the weights for positive utility (from gains). There are plenty of studies providing empirical evidence of sign-dependence, including Edwards (1953, 1954), Tversky and Kahneman (1992), Abdellaoui (2000), or Abdellaoui, l'Haridon et al. (2010).
} 
neither should the replacement of a loss by a different loss affect such midpoints; inconsistent midpoints are revealed only if a gain is replaced by a loss or vice versa. In a nutshell, our main preference tool requires that sign independence of revealed probability midpoints is violated once at the most, in which case we identify the location of the reference point.

In what follows, we present preliminary notation and formal expressions for the models of EU, RDU and PT (Sect. 2), informally introduce probability midpoints, and look at the distinct predictions for midpoints resulting from these models. In Sect. 3 we proceed by recalling the preference conditions shared by all three models. We highlight potential difficulties in deriving PT-foundations by giving examples of reference-dependent preferences that are similar to PT-preferences, which do not, in general, allow for the identification of both probability weighting functions. In the literature such preferences have hitherto been circumvented. In Sect. 4 we formally generalize the notion of probability midpoints and present our main preference condition and theorem for the case where the set of outcomes is finite. Extensions are discussed in Sect. 5. In particular, we allude to a procedure that shows how our probability midpoint tool can be employed to identify the location of reference points. The literature on reference points is growing, and different models and approaches have emerged; for instance, there are choice situations in which the reference point may not be a unique degenerate outcome as in PT; thus, a brief summary of this literature is in order. This is done in Sect. 6, where we also discuss issues related to midpoints, the central tool for our PT-foundation. Concluding remarks are in Sect. 7. The Appendix contains further elaborations and proofs.

\section{Preliminaries}

This section recalls the standard framework for decision under risk and the decision models of expected utility, rank-dependent utility and prospect theory, explaining how the second model extends the first through deviating from the linear treatment of probabilities and how the latter model extends the second through the reference point impacting the nonlinear probability treatment.

\subsection{Notation}

Let $X$ denote the nonempty set of outcomes. A prospect is a finite probability distribution over $X$. Prospects are labeled as $P=\left(p_{1}: x_{1}, \ldots, p_{n}: x_{n}\right)$ with the usual interpretation that outcome $x_{j} \in X$ is obtained with probability $p_{j}$, for $j=1, \ldots, n$. Naturally, $p_{j} \geq 0$ for each $j=1, \ldots, n$ and $\sum_{i=1}^{n} p_{i}=1$. Let $\mathcal{L}$ denote the set of all prospects.

A preference relation, denoted $\succcurlyeq$, is assumed over $\mathcal{L}$. Its restriction to subsets of $\mathcal{L}$ (e.g., all degenerate prospects where one of the outcomes is received for sure) is also denoted by $\succcurlyeq$. The symbol $\succcurlyeq$ means "weak preference" from which $\succ$ (strict preference) and $\sim$ (indifference) are defined as usual. The function $V$ represents (or is a representation of the preference $\succcurlyeq$ on $\mathcal{L}$, if $V$ assigns a real value to each prospect, such that for all $P, Q \in \mathcal{L}$ we have $P \succcurlyeq Q \Leftrightarrow V(P) \geq V(Q)$. This general 
representation $V$ will be required to satisfy several properties including those that reflect the behavior corresponding to the treatment of probabilities.

Next, we recall the functional expressions of expected utility, rank-dependent utility and prospect theory, which are specific representations of the preference $\succcurlyeq$ on $\mathcal{L}$. In all these models a utility function, $u$, for outcomes exists that is strictly monotonic (that is, $u: X \rightarrow \mathbb{R}$ satisfies $\left.u\left(x_{i}\right) \geq u\left(x_{j}\right) \Leftrightarrow x_{i} \succcurlyeq x_{j}\right)$. As a result, outcomes that are indifferent receive the same utility value. To simplify the exposition we henceforth assume, without loss of generality, that no two distinct outcomes in $X$ are indifferent. This allows us to strictly rank outcomes from best to worst within a prospect; this particular ordering of outcomes is meaningful in rank-dependent models.

\subsubsection{Expected utility}

Under expected utility (EU) prospects $P=\left(p_{1}: x_{1}, \ldots, p_{n}: x_{n}\right)$ are evaluated and compared using the representation

$$
\mathrm{EU}(P)=\sum_{j=1}^{n} p_{j} u\left(x_{j}\right)
$$

for a utility function, $u$, which assigns a real number to each outcome and is strictly monotone. Under EU the utility is cardinal, i.e., it is unique up to multiplication by a positive number $A$ and addition of a constant $B$. In EU the probabilities are treated linearly. This can be inferred from the "weight" $p_{j}$ corresponding to the utility for outcome $x_{j}$ in the above equation. The next model deviates from the linear treatment of probabilities.

\subsubsection{Rank-dependent utility}

The nonlinear treatment of probabilities in rank-dependent models is incorporated by making the relative position of outcomes meaningful for behavior. We can assume, without loss of generality, that outcomes within a prospect are ordered, as traditionally done, from best to worst in preference or rank-ordered; i.e., writing $P=\left(p_{1}\right.$ : $x_{1}, \ldots, p_{n}: x_{n}$ ) explicitly means that $x_{1} \succ \cdots \succ x_{n}$. Rank-dependent utility (RDU) holds if the preference is represented by

$$
\operatorname{RDU}(P)=\sum_{j=1}^{n}\left[w\left(p_{1}+\cdots+p_{j}\right)-w\left(p_{1}+\cdots+p_{j-1}\right)\right] u\left(x_{j}\right)
$$

where we use the convention that the $\operatorname{sum} \sum_{j=i}^{l} p_{j}=0$ for $l<i$. Utility in RDU is similar to EU. Additionally, RDU involves a weighting function, $w$, for (decumulative) probabilities that is uniquely determined. Formally, the weighting function is a mapping from the probability interval $[0,1]$ into $[0,1]$ that is strictly increasing with $w(0)=$ 
0 and $w(1)=1$. In this paper the axiomatically derived weighting functions are continuous on $[0,1] .^{4}$

The probability of obtaining a better-ranked outcome is referred to as good-news probability (formally called rank in Wakker 2010, Definition 5.4.1). In Eq. (2) the "decision weight" corresponding to the utility for outcome $x_{j}$ is the difference between transformed good-news probabilities. Denoting by $p_{j}^{d}:=\sum_{i=1}^{j} p_{i}$ the decumulative probability of $x_{j}, j=1, \ldots, n$, one can rewrite Eq. (2) as

$$
\operatorname{RDU}(P)=\sum_{j=1}^{n-1} w\left(p_{j}^{d}\right)\left[u\left(x_{j}\right)-u\left(x_{j+1}\right)\right]+u\left(x_{n}\right) .
$$

Clearly, having $w(p)=p$ for all probabilities $p$ in the latter equation implies EU. This confirms that $w$ under RDU captures deviations from linear treatment of good-news probabilities.

To simplify the subsequent exposition, we also present the expression for RDU, which uses the dual probability weighting function, $\hat{w}(p):=1-w(1-p)$ for all $p \in[0,1]$. The dual weighting function is defined over cumulative or bad-news probabilities. Then, we can rewrite RDU for $P$ as follows

$$
\operatorname{RDU}(P)=u\left(x_{1}\right)+\sum_{j=2}^{n} \hat{w}\left(p_{j}^{c}\right)\left[u\left(x_{j}\right)-u\left(x_{j-1}\right)\right]
$$

with transformed cumulative probabilities $p_{j}^{c}:=\sum_{i=j}^{n} p_{i}, j=1, \ldots, n$.

\subsubsection{Prospect theory}

The main model of interest in this paper extends RDU by incorporating referencedependence. That is, it assumes an outcome, $r \in X$, called the reference point, such that outcomes strictly preferred to it are gains and have a positive utility, and outcomes strictly dispreferred to $r$ are losses with a negative utility value. The treatment of probabilities depends on whether the latter are associated with gains or with losses. Specifically, under prospect theory (PT), a prospect $P=\left(p_{1}: x_{1}, \ldots, p_{n}: x_{n}\right)$ is evaluated according to the rank-ordering of outcomes and also according to the position of outcomes relative to the reference point. That is, there are two weighting functions $w^{+}$and $w^{-}$and a utility function for outcomes with $u(r)=0$, such that:

\footnotetext{
4 There is, however, empirical and theoretical interest in discontinuous weighting functions at 0 and at 1; see Kahneman and Tversky (1979), Birnbaum and Stegner (1981), Bell (1985), Cohen (1992), Wakker (1994, 2001), Chateauneuf et al. (2007), al-Nowaihi and Dhami (2010), Webb and Zank (2011), Andreoni and Sprenger (2010, 2012); we briefly discuss such potential extensions in Sect. 5. Webb (2017) suggests continuous extensions for a class of piecewise linear probability weighting functions that are empirically indistinguishable from those with discontinuities at 0 and 1 (see also Webb (2015) for event weighting under ambiguity).
} 
- if all outcomes in $P$ are (weakly) preferred to the reference point (i.e., we have no losses), then $P$ is evaluated by

$$
\mathrm{PT}(P)=\mathrm{RDU}^{+}(P) \text { using the weighting function } w^{+} \text {; }
$$

- if all outcomes in $P$ are (weakly) dispreferred to the reference point (i.e., we have no gains), then $P$ is evaluated by

$$
\mathrm{PT}(P)=\mathrm{RDU}^{-}(P) \text { using the weighting function } w^{-} \text {; }
$$

- if $P$ assigns positive probability to both gains and losses, then the PT-value of $P$ is the sum of $P$ 's gain and loss parts. That is, with $P^{+}$, the gain part of $P$, being the prospect " $P$ with all losses replaced by $r$ " and the loss part, $P^{-}$, being the prospect " $P$ with all gains replaced by $r$," the PT-value of $P$ is given by

$$
\operatorname{PT}(P)=\operatorname{PT}\left(P^{+}\right)+\operatorname{PT}\left(P^{-}\right)
$$

where the qualification $u(r)=0$ applies. It is custom for PT to express the treatment of probabilities for losses using the dual weighting function, $\hat{w}^{-}$. For instance, if $r=x_{k}$ for some $2 \leq k \leq n-1$, the PT-value of $P$ is

$$
\begin{aligned}
\operatorname{PT}(P)= & \sum_{j=1}^{k-1} w^{+}\left(p_{j}^{d}\right)\left[u\left(x_{j}\right)-u\left(x_{j+1}\right)\right] \\
& +\sum_{j=k+1}^{n} \hat{w}^{-}\left(p_{j}^{c}\right)\left[u\left(x_{j}\right)-u\left(x_{j-1}\right)\right] .
\end{aligned}
$$

From the preceding three cases one can infer that specific uniqueness results apply for PT. Indeed, if $X$ contains no losses (or if $X$ contains no gains), then RDU holds. Similarly, if $w^{+}=w^{-}$, PT reduces to an RDU-representation irrespective of the interpretation given to outcomes. In all of these cases we have a cardinal utility and a (single) uniquely determined weighting function capturing the treatment of probabilities. Thus, as the reference point is meaningless for that treatment so is the restriction $u(r)=0$. However, if $X$ contains gains and losses and $w^{+} \neq w^{-}$, we have two weighting functions that are uniquely determined; (only) in this case $u(r)=0$ must hold and, therefore, the utility is a ratio scale (i.e., it is unique up to multiplication by a positive constant). As a consequence, gains have positive utility and losses are assigned a negative utility value; hence, we formally refer to the condition $w^{+} \neq w^{-}$ as sign-dependence.

Except for Schmidt and Zank (2012), all existing foundations for PT assume the reference point as given from the outset; hence, also the uniqueness of the reference point is assumed. Here we drop the assumption of knowing the reference point in advance and we also dispense of structural assumptions for outcomes (e.g., requiring outcomes to be real valued) that are usually imposed for obtaining PT-foundations. 
Therefore, in our uniqueness results we explicitly state that for PT it is the signdependence that implies the uniqueness of the reference point. This is indeed the feature that distinguishes PT-preferences from RDU-preferences and, hence, from EUpreferences. The corollary of this observation is that, in principle, sign-dependence can be used to reveal the reference point from behavior. To do this, we employ preference conditions that build on revealed or elicited probability midpoints. Next, we present this tool, and we look at the implication of midpoints for the just presented models.

\subsection{Probability midpoints}

Probability midpoints are measurements revealed from preferences; they are derived using shifts in probability mass across outcomes. To motivate the midpoint concept, suppose we have two prospects $P, Q$ over outcomes in the set $\left\{x_{1}, x_{m}, x_{n}\right\} \subset X$, with the usual ranking $x_{1} \succ x_{m} \succ x_{n}$. Let

$$
P=\left(\alpha: x_{1}, 1-p-\alpha: x_{m}, p: x_{n}\right) \text { and } Q=\left(\beta: x_{1}, 1-q-\beta: x_{m}, q: x_{n}\right)
$$

be such that $\alpha<\beta$ and $P \sim Q$. (Similar arguments are used if $\beta<\alpha$.) The latter indifference indicates that the probability mass $\beta-\alpha$ for $x_{1}$ compensates for the difference between probabilities $p$ and $q$ for $x_{n}$ in the prospects $P$ and $Q$. This can be inferred from Fig. 1, which depicts the corresponding indifference sets in the probability triangle with outcomes $x_{1}, x_{m}$ and $x_{n}$, where probabilities of $x_{n}\left(x_{1}\right)$ are indicated on the horizontal (vertical) axis and the remaining probability is given to outcome $x_{m}$.

We now shift probability mass $\beta-\alpha$ from $x_{m}$ to $x_{1}$ in prospect $P$, which requires a joint shift of probability mass $\gamma-\beta$ from $x_{m}$ to $x_{1}$ in prospect $Q$ to obtain an indifference between the resulting prospects, i.e., we have

$$
P^{\prime}:=\left(\beta: x_{1}, 1-p-\beta: x_{m}, p: x_{n}\right) \sim\left(\gamma: x_{1}, 1-q-\gamma: x_{m}, q: x_{n}\right)=: Q^{\prime} .
$$

Considering the vertical axis, Fig. 1 illustrates that the "preference-distance" $\beta-\alpha$ between $P$ and $P^{\prime}$ is similar to the preference-distance $\gamma-\beta$ between $Q$ and $Q^{\prime}$.

Fig. 1 Elicited probability midpoint $\beta$

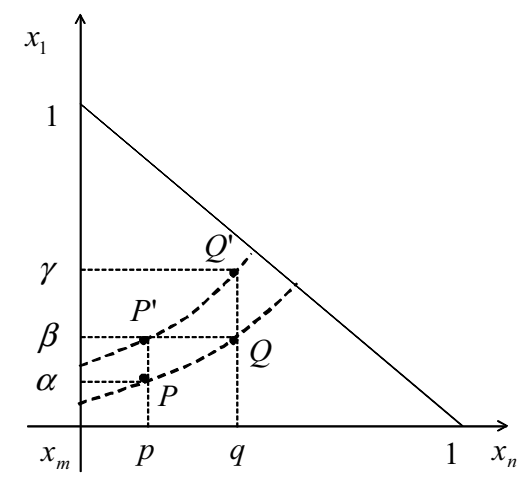


That is, substituting $\alpha$ for $\beta$ in $P$ leads to an improvement that is equivalent in terms of the preference to the improvement resulting from substituting $\beta$ for $\gamma$ in $Q$. Thus, in terms of the measured preference-distance, it means that $\beta$ is perceived half-way between $\alpha$ and $\gamma$ based on the probability-outcome pairs $\left(p: x_{n}\right)$ and $\left(q: x_{n}\right)$ in $P$ and $Q$, respectively, as gauges. We call $\beta$ a (revealed) probability midpoint between $\alpha$ and $\gamma$.

The representations introduced in the preceding subsection make specific predictions for probability midpoints. In particular, they all imply that probability midpoints are, with model-specific qualifications, independent of the gauges used to reveal those midpoints. We explore these implications next.

\subsubsection{Model-specific midpoints}

To illustrate the implications of midpoints for our models, consider EU first. Substitution of EU for the indifferences $P \sim Q$ and $P^{\prime} \sim Q^{\prime}$ used to reveal the probability midpoint $\beta$ in Fig. 1 implies, respectively,

$$
\begin{aligned}
\alpha u\left(x_{1}\right)+(1-p-\alpha) u\left(x_{m}\right)+p u\left(x_{n}\right) & =\beta u\left(x_{1}\right)+(1-q-\beta) u\left(x_{m}\right)+q u\left(x_{n}\right) \\
\text { and } \beta u\left(x_{1}\right)+(1-p-\beta) u\left(x_{m}\right)+p u\left(x_{n}\right) & =\gamma u\left(x_{1}\right)+(1-q-\gamma) u\left(x_{m}\right)+q u\left(x_{n}\right) .
\end{aligned}
$$

Taking the difference between the resulting two equations and cancelling common terms yields

$$
\beta=\frac{\alpha+\gamma}{2}
$$

proving that, beyond probability midpoints being independent of the outcomes used to elicit them, EU demands that $\beta$ is the arithmetic midpoint between $\alpha$ and $\gamma$. By contrast, RDU dispenses of such linearity in the treatment of probabilities, but maintains the independence of the revealed midpoint from outcomes. Similar to the preceding derivation, for RDU we obtain

$$
w(\beta)=\frac{w(\alpha)+w(\gamma)}{2}
$$

showing that, on the probability weighting scale, $\beta$ is perceived half-way between $\alpha$ and $\gamma$.

As with RDU, PT allows for nonlinear treatment of probabilities, but it marginally restricts the independence of probability midpoints from outcomes. Specifically, implementing the preceding elicitation of probability midpoints for the case that $x_{1}, x_{m}$ and $x_{n}$ are gains we obtain, similar to RDU,

$$
w^{+}(\beta)=\frac{w^{+}(\alpha)+w^{+}(\gamma)}{2} .
$$


The elicitation of midpoints for the case where the gains $\left\{x_{1}, x_{m}, x_{n}\right\}$ are replaced by a set of losses $\left\{y_{1}, y_{m}, y_{n}\right\}$ can be implemented using the same $\alpha$ and $\beta$ and initially identifying $\tilde{p}$ and $\tilde{q}$ such that

$$
\tilde{P}:=\left(\alpha: y_{1}, 1-\tilde{p}-\alpha: y_{m}, \tilde{p}: y_{n}\right) \sim\left(\beta: y_{1}, 1-\tilde{q}-\beta: y_{m}, \tilde{q}: y_{n}\right)=: \tilde{Q}
$$

and, subsequently, finding probability $\gamma^{*}$ such that

$$
\tilde{P}^{\prime}:=\left(\beta: y_{1}, 1-\tilde{p}-\beta: y_{m}, \tilde{p}: y_{n}\right) \sim\left(\gamma^{*}: y_{1}, 1-\tilde{q}-\gamma^{*}: y_{m}, \tilde{q}: y_{n}\right)=: \tilde{Q}^{\prime}
$$

Now, substitution of PT in the preceding two indifferences, taking differences of the implied equations and elimination of common terms, leads to

$$
w^{-}(\beta)=\frac{w^{-}(\alpha)+w^{-}\left(\gamma^{*}\right)}{2}
$$

In general, we have $\gamma \neq \gamma^{*}$ (unless we have sign independence under PT) and this inconsistency means that $\beta$, the probability midpoint of $\alpha$ and $\gamma$ for $w^{+}$, is not necessarily a midpoint of $\alpha$ and $\gamma$ for $w^{-}$. In the presence of sign-dependence it must be the case that (at least some) probability midpoints for gains are not identical to those for losses. It is indeed this observation that, in a reversed sense, is exploited to formulate preference conditions that identify the reference point. Before doing so, we recall the standard preference conditions that are shared by all the models considered above.

\section{Additive representation}

The decision models presented in the preceding section share several properties that, when combined, imply an additive representation over prospects. For our specific framework these properties of preferences have been presented before (e.g., in Zank 2010); corresponding properties for general rank-ordered sets were provided in Wakker (1993). For completeness we recall these properties here and summarize their implications for preferences in a lemma. This also allows us to highlight some potential difficulties that are particular to PT-preferences. Since it simplifies the exposition, we assume that the set of outcomes is finite, i.e., $X=\left\{x_{1}, \ldots, x_{n}\right\}$ for some natural number $n$. In Sect. 5 we indicate how our results can be extended to infinite sets of outcomes where, in contrast to the finite outcome case, preferences may be sign-dependent, but the reference point need not be included in the outcome set. Corresponding examples are then provided.

\subsection{Traditional preference conditions}

It is well known that, for the existence of a representing function for the preference, a necessary requirement is that the preference relation $\succcurlyeq$ is complete $(P \succcurlyeq Q$ or $P \preccurlyeq Q$ 
for all $P, Q \in \mathcal{L}$ ) and transitive; that is, the preference relation is a weak order. We summarize this property as our first axiom.

WEAK ORDER: The preference relation satisfies completeness and transitivity.

Further common properties for EU-, RDU- and PT-preferences are those of (firstorder stochastic) dominance and of continuity in probabilities. They are presented next. We say that $P$ first-order stochastically dominates $Q$ if $\sum_{x \in X} p_{x} \geq \sum_{x \in X} q_{x}$ and $P \neq Q$, where $p_{x}$ and $q_{x}$ refer to the probability that $P$, respectively, $Q$ assign to outcome $x \in X$.

DOMINANCE: The preference relation satisfies dominance (or monotonicity in decumulative probabilities) if $P \succ Q$ whenever $P$ first-order stochastically dominates $Q$.

Since Herstein and Milnor (1953), it is custom to regard $\mathcal{L}$ as a mixture space endowed with the operation of probability mixing. That is, for each probability $\mu \in$ $[0,1]$ and all prospects $P, Q \in \mathcal{L}$, the $\mu$-probability mixture of $P$ and $Q$, denoted $\mu P+(1-\mu) Q$, which assigns probability $\mu p_{x}+(1-\mu) q_{x}$ to outcome $x \in X$, is also a prospect in $\mathcal{L}$. We use this operation in the definition of continuity for the preference relation $\succcurlyeq$.

CONTINUITY: The preference relation $\succcurlyeq$ satisfies Jensen-continuity on the set of prospects $\mathcal{L}$ if for all prospects $P \succ Q$ and $R$ there exist $\rho, \mu \in(0,1)$ such that $\rho P+(1-\rho) R \succ Q$ and $P \succ \mu R+(1-\mu) Q$.

A monotonic weak order that satisfies Jensen-continuity on $\mathcal{L}$ also satisfies the stronger Euclidean-continuity on $\mathcal{L}$ (see, for example, Abdellaoui 2002, Lemma 18). Consequently, the three conditions taken together imply the existence of a continuous function $V: \mathcal{L} \rightarrow \mathbb{R}$, strictly monotonic in decumulative probabilities, that represents $\succcurlyeq$ on $\mathcal{L}$ (Debreu 1954). ${ }^{5}$ The next subsection provides a condition under which the representation $V$ is additively separable. We present it separately, as the strong formulation of the property implies EU and, thus, imposes a linear treatment of probabilities, while the weaker version allows more flexibility for the treatment of probabilities as required in RDU and PT.

\subsection{Additive separability properties}

This subsection presents an independence property that is shared by EU, RDU and PT. It is formulated as a preference condition involving common shifts of probability mass between outcomes. We have informally used shifts in probabilities when defining elicited probability midpoints. Probability shifts can be regarded as substitutions of an outcome with probability $\varepsilon$ by a different outcome with that very same probability. Given prospect $P=\left(p_{1}: x_{1}, \ldots, p_{n}: x_{n}\right)$ we denote the prospect resulting from a shift of probability $\varepsilon$ from outcome $x_{i}$ to outcome $x_{j}$ in $P$ as the prospect $\varepsilon_{i, j} P:=$

\footnotetext{
5 This function may be unbounded when the probability of $x_{n}$ approaches 0 or the probability of $x_{1}$ approaches 1 .
} 
$\left(p_{1}^{\prime}: x_{1}, \ldots, p_{n}^{\prime}: x_{n}\right)$, with $p_{i}^{\prime}=p_{i}-\varepsilon, p_{j}^{\prime}=p_{j}+\varepsilon$, and $p_{m}^{\prime}=p_{m}$ for $m \notin\{i, j\}$. Whenever we use this notation, it is implicitly assumed that $p_{i} \geq \varepsilon>0$ to ensure that $\varepsilon_{i, j} P$ is a well-defined prospect in $\mathcal{L}$.

In terms of the indifference sets of the preference, these substitutions mean that, following a probability shift, a prospect is moved from one indifference set to a different one. We are interested in the effect of such substitutions for prospects that are on the same indifference set. For example, EU satisfies independence of the preferences under common probability shifts, meaning that indifference sets of a preference are mapped into new indifference sets when common probability shifts are applied. This property was termed "replacement invariance" in Machina (1989) and constitutes a sure thing principle for risky prospects similar to Savage (1954) sure thing principle for uncertainty. We adopt the latter terminology for the next axiom.

SURE THING PRINCIPLE FOR RISK: The preference relation $\succcurlyeq$ satisfies the sure thing principle (STP) for risk if

$$
P \succcurlyeq Q \Leftrightarrow \varepsilon_{i, i+1} P \succcurlyeq \varepsilon_{i, i+1} Q,
$$

whenever $P, Q, \varepsilon_{i, i+1} P, \varepsilon_{i, i+1} Q \in \mathcal{L}$.

STP is necessary for expected utility; it is also sufficient for EU in the presence of weak order, first-order stochastic dominance and Jensen-continuity (see, for example, Webb and Zank 2011, Theorem 5). The common consequence effect of Allais (1953) constitutes a violation of the sure thing principle for risk. Accordingly, RDU and PT, which both can accommodate the Allais paradox, satisfy a restricted version of the principle:

COMONOTONIC STP: The preference relation $\succcurlyeq$ satisfies the comonotonic sure thing principle (CSTP) for risk if

$$
P \succcurlyeq Q \Leftrightarrow \varepsilon_{i, i+1} P \succcurlyeq \varepsilon_{i, i+1} Q
$$

whenever $P, Q, \varepsilon_{i, i+1} P, \varepsilon_{i, i+1} Q \in \mathcal{L}$ are such that $p_{i}^{d}=q_{i}^{d}$.

CSTP says that common probability shifts maintain the preference between two prospects if the two prospects offer identical good-news probabilities for $x_{i-1}$. That is, the probability of obtaining $x_{i}$, the outcome from which probability is shifted away, or a better outcome is the same in both prospects. This is equivalent to saying that the cumulative probability of obtaining $x_{i+1}$ or a worse outcome is the same in both prospects, so they have identical bad-news probabilities too. Hence, a shift of a common probability in $P$ and $Q$ results in new prospects where the outcome $x_{i}$ is also of common rank. If one writes prospects as (de)cumulative distributions over $X$, one immediately observes that CSTP translates into an independence requirement on a rank-ordered or comonotonic set of cumulated distributions, hence the name for this property.

When CSTP is combined with the preference conditions in the preceding subsection, it implies an additive separability property across outcomes for the representing function $V$. The result, formally stated here, generally applies to the preference 
restricted to all prospects except perhaps the best prospect $\left(x_{1}\right)$ and the worst prospect $\left(x_{n}\right)$; the proof follows from Wakker's (1993, Theorem 3.2) result for additive representations on comonotonic sets.

Lemma 1 The following two statements are equivalent for a preference relation $\succcurlyeq$ on $\mathcal{L}$ :

(i) The preference relation $\succcurlyeq$ on $\mathcal{L} \backslash\left\{x_{1}, x_{n}\right\}$ is represented by an additive function

$$
V(P)=\sum_{j=1}^{n-1} V_{j}\left(p_{j}^{d}\right),
$$

with strictly increasing functions $V_{1}, \ldots, V_{n-1}:[0,1] \rightarrow \mathbb{R}$ which are continuous and bounded with the exception of $V_{1}$ and $V_{n-1}$, which could be unbounded at 1 and at 0 , respectively. ${ }^{6}$

(ii) The preference relation $\succcurlyeq$ is a Jensen-continuous weak order that satisfies dominance and the comonotonic sure thing principle for risk.

The functions $V_{1}, \ldots, V_{n-1}$ are jointly cardinal; that is, they are unique up to multiplication by a common positive constant and addition of a real number. The representation can continuously be extended to hold on $\mathcal{L}$ if $V_{1}$ is bounded from above and $V_{n-1}$ is bounded from below.

As shown in Wakker (1993, Proposition 3.5), by adding further preference conditions that imply a separation of the treatment of probabilities from the utility value assigned to outcomes, one can generally obtain boundedness of all functions in Lemma 1. This has been exploited, for instance, in the RDU-derivations in Diecidue et al. (2009) and in Webb and Zank (2011). For PT this also applies, provided that there are at least two gains and two losses, whence proportionality arguments can be exploited (see our Theorem 2). As we do not make assumptions about which outcome is the reference point, we cannot, in general, invoke information about the number of gains and losses from the outset. This means that the potential unboundedness reported in Lemma 1 cannot a priori be excluded. Consequently, this leads to difficulties for deriving a standard PT-representation, as we discuss next.

\subsection{Extended prospect theory}

Based on the additive representation of Lemma 1, in this subsection we further explore the consequences for obtaining PT-foundations when one of $V_{1}$ or $V_{n-1}$ is unbounded. In general, such unboundedness precludes the identification of probability weighting functions as is required for PT. Our main result shows that we are able to obtain representations for "extended PT-preferences" where a reference point can nonetheless be identified and, further, a utility for losses and a corresponding probability weighting function (if $V_{1}$ is unbounded), or a utility for gains with the associated probability

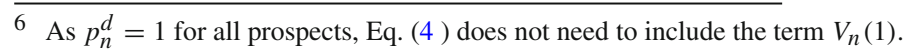


weighting function (if $V_{n-1}$ is unbounded), can still be derived. In particular, at most one of $V_{1}$ or $V_{n-1}$ can be unbounded. We provide specific examples to illustrate these extreme cases as they highlight specific types of preferences that are plausible when general outcome sets and reference-dependence are jointly considered. In the foundational literature on PT such behavior has hitherto been excluded due to the structural requirements on the set outcomes and the properties reflected in the corresponding utility functions.

Example 1 Assume that the representation of Lemma 1 holds for $X=\left\{x_{1}, x_{2}, x_{3}\right\}$ with $x_{1} \succ x_{2} \succ x_{3}$ and that $V_{1}$ and $V_{2}$ are bounded. Then, the representation is a PT-functional with $r=x_{2}$ as the reference point, unless $\succcurlyeq$ is represented by RDU.

The derivation of the PT-representation in Example 1 proceeds as follows. As $V_{1}$ and $V_{2}$ are jointly cardinal, we can pick the representation of preferences with $V_{1}(0)=0=V_{2}(1)$. By setting $w^{+}(p):=V_{1}(p) / V_{1}(1)$ for all $p \in[0,1]$ and $u\left(x_{1}\right):=V_{1}(1), \hat{w}^{-}(p):=1-V_{2}(p) / V_{2}(0)$ for all $p \in[0,1]$ and $u\left(x_{3}\right):=V_{2}(0)$, and further $u\left(x_{2}\right):=0$, we obtain

$$
V\left(p_{1}: x_{1}, p_{2}: x_{2}, p_{3}: x_{3}\right)=w^{+}\left(p_{1}\right) u\left(x_{1}\right)+\hat{w}^{-}\left(p_{3}\right) u\left(x_{3}\right)
$$

which is a PT-representation with reference point $r=x_{2}$.

If $w^{+}(p)=w^{-}(p)$ for all $p \in[0,1]$, one can show that Eq. (5) reduces to an RDUrepresentation with the corresponding uniqueness results. For the case that $w^{+} \neq w^{-}$, one can show that the values $V_{1}(0)$ and $V_{2}(1)$ are constants that are immaterial for the ranking of prospects, such that the representation in Eq. (5) is indeed PT with the corresponding uniqueness results; this aspect is further elaborated on in the Appendix.

For the case of Example 1, we are not aware of a preference condition, that explicitly identifies $x_{2}$ as a reference point in a manner that pins down PT with a ratio-scale utility, beyond the properties that imply additive separability as required in Lemma $1 .^{7}$ More generally, we observe that it is only in the case of $n=3$ that a reference point may exist, but that both $V_{1}$ and $V_{n-1}\left(=V_{2}\right)$ in Lemma 1 are unbounded. Such unboundedness precludes a separation of utility and sign-dependent probability weighting functions. Including such representations under a general notion of PT-preferences means that Lemma 1 already gives necessary and sufficient conditions for a corresponding representation. Given this observation, from here onward we require that $X$ has at least four strictly rank-ordered outcomes. The next two examples show that the assumption of more than three outcomes restricts, but does not eliminate unboundedness for a representation in the presence of reference-dependence.

Example 2 Consider the case that $n \geq 4$ and that the preference on $\mathcal{L} \backslash\left\{x_{1}\right\}$ is represented by

\footnotetext{
7 A more powerful result is provided in Abdellaoui (2002), who proposes behavioral conditions that ensure proportionality of the functions $V_{1}$ and $V_{2}$, thereby obtaining RDU where sign independence holds (see also Chateauneuf 1999).
} 


$$
W(P)=V_{1}\left(p_{1}\right)+\sum_{j=3}^{n} \hat{w}^{-}\left(p_{j}^{c}\right)\left[u\left(x_{j}\right)-u\left(x_{j-1}\right)\right],
$$

where $u$ and $\hat{w}^{-}$are as in PT with $r=x_{2}$ and $V_{1}\left(p_{1}\right)$ converges to $\infty$ when $p_{1}$ approaches 1, e.g., $V_{1}\left(p_{1}\right)=\left[\frac{p_{1}}{1-p_{1}}\right] u\left(x_{1}\right)$ for some positive number $u\left(x_{1}\right)$.

One can regard the preference in Example 2 as that of a patient who has been diagnosed with a severe disease, such as cancer. Suppose some potential medical interventions can lead to a range of outcomes, the best being $x_{1}=$ "fully cured from cancer," while other interventions may prolong life duration but do not offer positive probability for $x_{1}$. It is conceivable that the latter interventions are all perceived as leading to losses and, hence, they are perceived unattractive relative to an intervention with a positive probability for $x_{1}$. A related example is documented in Thaler and Johnson (1990) and analyzed in Barberis et al. (2001). After having faced a series of losses, many investors attempt to break even by engaging in very risky trades, despite the chances of breaking even being relatively small. Such investors appear to perceive the event of breaking even as leading to an extremely attractive outcome, while the complementary event is viewed as leading to losses of a tolerable magnitude in utility terms. The counterpart of Example 2 is the following one.

Example 3 Consider the case that $n \geq 4$ and that the preference on $\mathcal{L} \backslash\left\{x_{n}\right\}$ is represented by

$$
\bar{W}(P)=\sum_{j=1}^{n-2} w^{+}\left(p_{j}^{d}\right)\left[u\left(x_{j}\right)-u\left(x_{j+1}\right)\right]+V_{n-1}\left(1-p_{n}\right)
$$

where $u$ and $w^{+}$are as in PT with $r=x_{n-1}$ and $V_{n-1}$ is as in Lemma 1 and converging to $-\infty$ at 0 . Specifically, consider $V_{n-1}\left(1-p_{n}\right)=\left[\frac{p_{n}}{1-p_{n}}\right] u\left(x_{n}\right)$ for some negative number $u\left(x_{n}\right)$.

The functional in Example 3 can be thought of as a representation for a preference with an extreme form of aversion or pessimism, where the possible loss $x_{n}$ is extremely unattractive (e.g., ruin) and any prospect with a positive probability for $x_{n}$ will be regarded inferior to a prospect with zero probability for $x_{n}$. Individuals exhibiting this form of pessimism are willing to buy insurance at prices far above the actuarially fair value to completely avoid the loss $x_{n}$. Such behavior is reported in relation to substantially increased demand for flood and earthquake insurance after a corresponding event has occurred (Kunreuther et al. 1978; Palm 1995) ${ }^{8}$ and in the willingness to pay for the complete elimination of risk associated with a hazardous product (Viscusi et al. 1987).

Having elaborated on potential issues for deriving PT when the set of outcomes includes a single gain or a single loss, we proceed by keeping in mind that in such

8 Etner and Jeleva (2014) explain underinvestment in prevention schemes through the treatment of probabilities as captured in RDU. 
special cases extreme sensitivity in probabilities of best or worst outcomes may preclude a derivation of PT in which both probability weighting functions are uniquely specified. Instead, we may obtain what we term extended prospect theory: Preferences are either represented by PT on $\mathcal{L}$, or they are represented on $\mathcal{L} \backslash\left\{x_{1}\right\}$ by the functional in Eq. (6) with $V_{1}:[0,1) \rightarrow[0, \infty)$ unbounded at $p=1$, or they are represented on $\mathcal{L} \backslash\left\{x_{n}\right\}$ by Eq. (7) with $V_{n-1}:(0,1] \rightarrow(-\infty, 0]$ unbounded at $p=0$. As the preference conditions presented in the next section show, in such cases we can still identify the reference point from behavior if preferences are reference-dependent.

\section{Consistent probability midpoints}

We have already established that for EU-preferences probability midpoints are algebraic midpoints, that they are independent of outcomes and, hence, reference point independent. Similarly, RDU-preferences imply reference independence although, due to flexibility in the treatment of probabilities, RDU allows probability midpoints to differ from algebraic midpoints. PT-preferences are different. They allow for elicited probability midpoints to depend on the type (i.e., gain or loss), but not the magnitude of outcomes.

This section introduces consistency conditions for probability midpoints which, when added to statement (ii) of Lemma 1, allow for the identification of a reference point. To this aim, it is instructive to explore the implications of elicited probability midpoints for the preference representation in Lemma 1. Recall the indifferences $P \sim Q$ and $P^{\prime} \sim Q^{\prime}$ in Fig. 1. After substitution of the additive representation, subtracting the first equation from the second and cancelling common terms, we obtain $V_{1}(\beta)-V_{1}(\alpha)=V_{1}(\gamma)-V_{1}(\beta)$ or, equivalently,

$$
V_{1}(\beta)=\frac{V_{1}(\gamma)+V_{1}(\alpha)}{2}
$$

In terms of $V_{1}$, which captures the treatment of probabilities associated with outcome $x_{1}$, Eq. (8) states that $\beta$ is a $V_{1}$-midpoint between $\alpha$ and $\gamma$. The properties of the additive representation imply that locally one can always find midpoints and that the latter do not depend on other outcome stimuli. For instance, taking an outcome $x_{m^{\prime}} \neq x_{m}$ such that $x_{1} \succ x_{m^{\prime}} \succ x_{n}$ and considering preferences among prospects over the set $\left\{x_{1}, x_{m^{\prime}}, x_{n}\right\} \subset X$, one can find probabilities $\bar{p}, \bar{q}$ such that $\bar{P}=\left(\alpha: x_{1}, 1-\bar{p}-\alpha\right.$ : $\left.x_{m^{\prime}}, \bar{p}: x_{n}\right) \sim\left(\beta: x_{1}, 1-\bar{q}-\beta: x_{m^{\prime}}, \bar{q}: x_{n}\right)=\bar{Q}$. Now, shifting $\beta-\alpha$ from $x_{m^{\prime}}$ to $x_{1}$ in $\bar{P}$ requires an equivalent shift of $\bar{\gamma}-\beta$ from $x_{m^{\prime}}$ to $x_{1}$ in $\bar{Q}$ to obtain a new indifference, say $\bar{P}^{\prime} \sim \bar{Q}^{\prime}$. As before, substitution of the representation in Lemma 1 for the indifferences $\bar{P} \sim \bar{Q}$ and $\bar{P}^{\prime} \sim \bar{Q}^{\prime}$, subtraction of the resulting equations and cancellation of common terms, gives

$$
V_{1}(\beta)=\frac{V_{1}(\bar{\gamma})+V_{1}(\alpha)}{2}
$$

which can only hold if $\bar{\gamma}=\gamma$, for otherwise Eq. (8) is violated. 
The preceding analysis shows that revealed probability midpoints elicited through shifts of probability mass to $x_{1}$ are meaningful for $V_{1}$, i.e., they are consistent and independent of stimuli other than $x_{1}$. Similarly, midpoints for each function $V_{j}, j=2, \ldots, n-1$ can be elicited and are meaningful concepts given the additive separable representation of Lemma 1 . Next we present properties that demand further consistency for elicited probability midpoints.

\subsection{Good-news and bad-news midpoint consistency}

The properties presented next demand that probability midpoints derived from probability shifts to best (worst) outcomes are also midpoints when the same probability mass is shifted to outcomes of intermediate rank. These behavioral qualifications for preferences are referred to as consistency properties for probability midpoints.

Good- News Midpoint Consistency: For $m \in\{2, \ldots, n-1\}$, the preference relation $\succcurlyeq$ satisfies good-news midpoint consistency (GMC) above $x_{m}$, if for $P=$ $\left(\alpha: x_{1}, p_{m}-\alpha: x_{m}, p_{m+1}: x_{m+1}, \ldots, p_{n}: x_{n}\right)$ and $Q=\left(\beta: x_{1}, q_{m}-\beta:\right.$ $\left.x_{m}, q_{m+1}: x_{m+1}, \ldots, q_{n}: x_{n}\right)$ we have

$$
P \sim Q \&(\beta-\alpha)_{m, 1} P \sim(\gamma-\beta)_{m, 1} Q \Rightarrow(\beta-\alpha)_{m, \tilde{m}} P \sim(\gamma-\beta)_{m, \tilde{m}} Q
$$

for all $\tilde{m} \in\{1, \ldots, m\}$ whenever $\alpha \leq \beta \leq \gamma$ are probabilities such that $P, Q$, $(\beta-\alpha)_{m, 1} P$, and $(\gamma-\beta)_{m, 1} Q$ are from $\mathcal{L}{ }^{9}$

It is worth contrasting the normative content of GMC with that of the more restrictive von Neumann and Morgenstern independence axiom. In GMC, the probability mass $\beta-\alpha$ in $(\beta-\alpha)_{m, 1} P$ is additional good-news probability for $x_{1}$ relative to $P$. Based on preferences, this improvement is equivalent to the additional good-news probability $\gamma-\beta$ in $(\gamma-\beta)_{m, 1} Q$ as compared to $Q$. GMC requires that such good-news probabilities are of equivalent value for preferences when shifted to a common outcome $x_{\tilde{m}}$ (i.e., a degenerate prospect) that is ranked between $x_{1}$ and $x_{m}$. The latter aspect indicates that preferences may be sensitive to the rank-ordering of outcomes when shifting equivalent good-news probabilities. Such rank-ordering restrictions do not apply to the von Neumann and Morgenstern independence axiom where probability mass is taken away proportionally from each outcome and shifted to another common, and not necessarily degenerate, prospect $S$ :

$$
P \sim Q \Leftrightarrow \rho S+(1-\rho) P \sim \rho S+(1-\rho) Q
$$

for all $\rho \in[0,1]$ and all $P, Q, S \in \mathcal{L}{ }^{10}$ Clearly, as no further restrictions are placed on the "common risk" type $S$, the latter property applies more generally than GMC

\footnotetext{
9 In this definition we could have included the cases $m=1$ and $m=n$ as the property then trivially holds.

10 In the literature different formulations of the von Neumann and Morgenstern independence axiom have been used, e.g., $P \succcurlyeq Q \Leftrightarrow \rho S+(1-\rho) P \succcurlyeq \rho S+(1-\rho) Q$ for all $P, Q, S \in \mathcal{L}$ and all $\rho \in[0,1]$. The definition presented here is equivalent given the employed weak order, continuity and dominance properties of $\succcurlyeq$.
} 
and is, thus, more powerful. In particular, the independence axiom implies that elicited probability midpoints are also algebraic midpoints, the characteristic property of EUpreferences.

It can be verified that RDU satisfies GMC above $x_{m}$ for all $m=2, \ldots, n-1$. This has been shown in Zank (2010). Similarly, this holds for the preference with the representation of Example 3. The next lemma shows that PT satisfies GMC above $x_{m}$ if all outcomes above $x_{m}$ are gains, hence, in particular if $x_{m}$ is the reference point.

Lemma 2 Assume that the preference $\succcurlyeq$ on $\mathcal{L}$ is represented by $P T$ (or by extended PT on $\left.\mathcal{L} \backslash\left\{x_{n}\right\}\right)$. Then $\succcurlyeq$ satisfies good-news midpoint consistency above $x$ whenever all outcomes ranked above $x$ are gains.

The next property is the dual analog of GMC as it focuses on shifting equivalent probability mass to outcomes of lower rank.

BAD- News Midpoint Consistency: For $m \in\{2, \ldots, n-1\}$, the preference relation $\succcurlyeq$ satisfies bad-news midpoint consistency (BMC) below $x_{m}$, if for $P=$ $\left(p_{1}: x_{1}, \ldots, p_{m-1}: x_{m-1}, p_{m}-\alpha: x_{m}, \alpha: x_{n}\right)$ and $Q=\left(q_{1}: x_{1}, \ldots, q_{m-1}:\right.$ $\left.x_{m-1}, q_{m}-\beta: x_{m}, \beta: x_{n}\right)$ we have

$$
P \sim Q \&(\beta-\alpha)_{m, n} P \sim(\gamma-\beta)_{m, n} Q \Rightarrow(\beta-\alpha)_{m, \tilde{m}} P \sim(\gamma-\beta)_{m, \tilde{m}} Q,
$$

for all $\tilde{m} \in\{m, \ldots, n\}$ whenever $\alpha \leq \beta \leq \gamma$ are probabilities such that $P, Q,(\beta-$ $\alpha)_{m, n} P$, and $(\gamma-\beta)_{m, n} Q$ are from $\mathcal{L}$.

The preference represented by the function in Example 2 satisfies BMC below $x_{m}$ for all $m \in\{2, \ldots, n-1\}$. Likewise, RDU-preferences satisfy BMC below each outcome. The next lemma confirms that PT satisfies BMC below all outcomes which are not gains, that is, below all losses and below the reference point.

Lemma 3 Assume that the preference $\succcurlyeq$ on $\mathcal{L}$ is represented by $P T$ (or by extended PT on $\left.\mathcal{L} \backslash\left\{x_{1}\right\}\right)$. Then $\succcurlyeq$ satisfies bad-news midpoint consistency below $x$ whenever all outcomes ranked below $x$ are losses.

If we have reference-dependence and we know the location of the reference point, then it is easy to formulate a sign-dependent midpoint consistency condition that characterizes PT when combined with the properties in Lemma 1. If there are two or more gains and two or more losses, all we need is GMC above all outcomes that are not losses and BMC below all outcomes that are not gains. Although not stated formally, this result is new and provides PT-foundations for general sets of outcomes, thereby directly extending the RDU foundations of Nakamura (1995), Abdellaoui (2002), Abdellaoui and Wakker (2005), and Zank (2010). In the case that there is one gain or one loss, we further need to assume that the additive representation consists of bounded functions; alternatively, the domain of the preference is limited to all but the best and worst prospects, in which case we obtain foundations for extended PT. Next we proceed without assuming that we know the location of the reference point. 


\subsection{Reference point revealing midpoint consistency}

In general, we do not know which of the outcomes in $X$ is the reference point. To obtain PT-foundations we develop a technique to first reveal the reference point before invoking the appropriate midpoint consistency properties. For instance, we can take outcomes in turn and verify if probability midpoints are independent of the outcomes used to reveal those midpoints. For PT-preferences a violation of this test of midpoint consistency is allowed once at the most. By starting with probability midpoint elicitations at best (worst) outcomes and moving sequentially toward lower (better)-ranked outcomes, we can test for "probability midpoint consistency" for all outcomes. Having finitely many outcomes means that this process will terminate with either universal consistency (i.e., there is no reference point affecting preferences) or a single violation (i.e., there exists a reference point that affects preferences). Observing multiple violations of consistency means that PT cannot hold. While this will be excluded by our main preference condition, it is clear that our consistency test can also be used to falsify PT. Applying the consistency property repeatedly comes down to an algorithmic procedure (see also Sect. 5.3) that allows us to identify outcomes that are gains and outcomes that are losses if preferences are reference-dependent. This motivates the following property. ${ }^{11}$

ReFerence Point REvealing MidPoint Consistency: The preference relation $\succcurlyeq$ satisfies reference point revealing midpoint consistency (RMC) if for each $m \in\{2, \ldots, n-1\}$ the preference satisfies good-news midpoint consistency above $x_{m}$ or bad-news midpoint consistency below $x_{m-1}$ (or both).

Before turning to the main result, we elaborate further on some implications of RMC. Suppose that $m_{\max } \in\{2, \ldots, n-1\}$ is the largest index such that GMC above $x_{m_{\max }}$ holds. Then, in the presence of an additive representation, by repeated application of GMC, one can show that GMC above $x_{j}$ holds for all $j \leq m_{\max }$, that is, for all outcomes ranked above $x_{m_{\max }}$. Similarly, if $m_{\min } \in\{2, \ldots, n-1\}$ is the smallest index such that BMC below $x_{m_{\min }-1}$ holds, then BMC below $x_{j}$ holds for all $j \geq m_{\min }-1$ in the presence of an additive representation. Therefore, as RMC demands for each $m \in\{2, \ldots, n-1\}$ that GMC above $x_{m}$ or BMC below $x_{m-1}$ holds, either $m_{\max }=$ $m_{\min }=k$, i.e., a single index $k \in\{2, \ldots, n-1\}$ exists such that GMC above $x_{k}$ or BMC below $x_{k-1}$ holds, or (as shown in the Appendix) for all $m \in\{2, \ldots, n-1\}$ both GMC above $x_{m}$ and BMC below $x_{m-1}$ hold. In the latter case we have sign independence, while in the former case we have sign-dependence and $r=x_{k}$ is the unique reference point. Under each of these conclusions one can derive (extended) PT. We conclude this section by summarizing this result.

Theorem 1 The following two statements are equivalent for a preference relation $\succcurlyeq$ on $\mathcal{L}$ :

(i) The preference relation $\succcurlyeq$ is represented by extended PT.

11 The idea of formulating preference conditions such that they imply the existence of an outcome where behavior changes suddenly has also been used in Schmidt and Zank (2012). 
(ii) The preference relation $\succcurlyeq$ is a Jensen-continuous weak order that satisfies dominance, the comonotonic sure thing principle for risk and reference point revealing midpoint consistency.

Where they exist, the probability weighting functions are uniquely determined. Further, if $w^{+}=w^{-}$, utility is cardinal as preferences agree with rank-dependent utility; otherwise, the utility function is a ratio scale and there is a unique index $k \in\{2, \ldots, n-1\}$ such that the reference point is $r=x_{k}$.

The proof of the preceding theorem is in the Appendix. The next section looks at extensions of our main result.

\section{Extensions}

In the previous sections we have assumed that no outcomes are indifferent. This requirement can be relaxed if there are at least four strictly ordered outcomes in the finite set $X$. All results remain valid if we restrict the analysis to the set of representatives for each indifference set of outcomes; within an indifference set all outcomes have the same utility value. As pointed out in Sect. 3, our results also remain valid if we include the case that there are exactly three strictly ordered outcomes, however, then RMC trivially holds and, given Lemma 1 with boundedness conditions for the additive representation satisfied, PT follows as indicated in Example 1. If boundedness conditions do not hold one must allow for extended PT-representations as suggested in Examples 2 and 3 , where the utility of the single gain, respectively, the single loss and the corresponding probability weighting function cannot be identified separately as the representing functions exhibit asymptotic behavior when approaching extreme probabilities. Such behavior is excluded if preferences agree with RDU, the special case of PT with sign independence. Finally, we recall that, in the trivial case of having at most two strictly ordered outcomes, the dominance property ensures the existence of an ordinal representation; it is well known that there is insufficient structure on the set of prospects to obtain more meaningful results for the two-outcome case.

In our derivation of PT it has been essential that the weighting functions are continuous at 0 and at 1 . Discontinuities at these extreme probabilities are, however, empirically meaningful. We could adopt a weaker version of continuity for probabilities that is restricted to prospects that have common best and worst outcomes each with a positive objective probability. Such conditions have been used in Cohen (1992) and more recently in Webb and Zank (2011) where probability weighting functions are derived that are linear and discontinuous at extreme probabilities. These weighting functions can then be described by two parameters. As Webb and Zank show, this relaxation of continuity in probabilities comes at a price. They require additional structural assumptions for the preference in order to obtain consistency of those parameters across sets of prospects with different worst and best outcomes. Also, specific consistency principles that imply the uniqueness of those parameters are required. We conjecture that in our framework such consistency principles can be formulated for nonlinear weighting functions that are discontinuous at 0 and at 1 . A formal derivation of PT with such weighting functions is, however, beyond the scope of this paper. 


\subsection{Omitting asymptotic behavior}

In this subsection we provide a variant of the probability midpoint condition that excludes unboundedness of the functions $V_{1}$ and $V_{n-1}$ in Theorem 1. If signdependence holds, the property can be seen as a condition that imposes the existence of at least two gains and at least two losses. With a further outcome that acts as the reference point, this property therefore assumes at least five strictly ordered outcomes. Under this mild richness assumption for outcomes, the following property demands consistent probability midpoints at the two best outcomes and, separately, consistent midpoints at the two worst outcomes and, additionally, RMC.

BOUNDED RMC: The preference relation $\succcurlyeq$ satisfies bounded $R M C(b-R M C)$ if RMC holds and further GMC holds above $x_{3}$ and BMC holds below $x_{n-2}$.

The augmented b-RMC property uses the information that the two best outcomes are of the same type (both are gains) and that the two worst outcomes are also of the same type (they are losses). Such information is not usually available to the analyst, although in some cases such assumptions can plausibly be made. For instance, when a decision maker wishes to sell a good at an auction, there might be several low prices at which selling would definitely be perceived as making a loss (being substantially below the reservation price), while there might be several high prices at which selling the good is perceived as a definite gain.

If the set of outcomes is too small, declaring some outcomes as gains and some as losses may in fact pin down the model. For example, it should be clear that if there are only four strictly rank-ordered outcomes in $X$, then b-RMC implies that GMC holds above $x_{3}$ and BMC holds below $x_{2}$. For the additive representation in Lemma 1 this has the implication that probability midpoints for $V_{1}$ are also midpoints for $V_{2}$ (inferred from GMC above $x_{3}$ ) and probability midpoints for $V_{3}$ are also midpoints for $V_{2}$ (inferred from BMC below $x_{2}$ ). Hence, midpoints are consistent throughout and this excludes sign-dependence; hence, RDU is implied.

The next result explicitly assumes that there are at least five strictly ordered outcomes, to allow for reference-dependence when b-RMC is invoked.

Theorem 2 Assume that the finite set of outcomes $X$ has at least five strictly ranked outcomes. The following two statements are equivalent for a preference relation $\succcurlyeq$ on $\mathcal{L}:$

(i) The preference relation $\succcurlyeq$ is represented by PT with either sign-dependence (in which case the reference point is $r=x_{k}$ for some $k \in\{3, \ldots, n-2\}$ ) or sign independence (RDU holds).

(ii) The preference relation $\succcurlyeq$ is a Jensen-continuous weak order that satisfies dominance, the comonotonic sure thing principle for risk and bounded reference point revealing midpoint consistency.

The probability weighting functions are uniquely determined. Further, if $w^{+}=w^{-}$, utility is cardinal as preferences agree with rank-dependent utility; otherwise, the utility function is a ratio scale and the reference point is unique.

The proof of the preceding result is in the Appendix. In Theorem 2 we exploit the fact that the set of outcomes is finite. If $X$ is infinite a property like b-RMC cannot 
simply be extended because, for example, for uncountable sets identifying the two best outcomes or the two worst outcomes may not be possible. But RMC can be formulated to hold on specific subsets of prospects as our next subsection shows. We now proceed to the discussion of how to obtain extended PT-foundations for infinite outcome sets.

\subsection{Infinite outcome sets}

As in the preceding sections, we restrict our analysis to the case that there are no indifferent outcomes. When $X$ is infinite some of our properties need to be extended. Weak order, continuity and dominance are as before. CSTP is invoked for each restriction of the preference to sets of prospects over finitely many common outcomes. For a finite set of outcomes $Y \subset X$ denote by $\mathcal{L}_{Y}$ the set of prospects that give zero probability to outcomes not contained in $Y$. We now formulate the extension of CSTP.

COMONOTONIC STP: The preference relation $\succcurlyeq$ satisfies the comonotonic sure thing principle $(C S T P)$ on $\mathcal{L}$ if $\succcurlyeq$ satisfies CSTP on $\mathcal{L}_{Y}$ for all finite sets $Y \subset X$.

One implication of (extended) PT is that, if the preference is restricted to prospects over a finite set of outcomes that contains only gains or the reference point, call it $X_{g}$ ( $g$ stands for good outcomes), then RDU represents that restriction of the preference. Similarly, RDU represents the preference restricted to prospects over a finite set of outcomes that contains only losses or the reference point, say $X_{b}$ ( $b$ referring to bad outcomes). The two RDU functions need not have the same probability weighting function; in particular, sign-dependence requires distinct weighting functions for the probabilities of gains and losses. Then, for prospects over $\mathcal{L}_{X_{g} \cup X_{b}}$, RMC will be satisfied only when the reference point is included in the union of the sets $X_{g}$ and $X_{b}$. For infinite sets of outcomes we, therefore, propose the following extension of RMC.

EXTENDED RMC: The preference relation $\succcurlyeq$ satisfies extended reference point revealing midpoint consistency (e-RMC) on $\mathcal{L}$ if for each outcome $x \in X$ one (or both) of the following statements apply:

(a) For each finite set $Y$ with all outcomes ranked above $x$ the preference $\succcurlyeq$ restricted to $\mathcal{L}_{Y \cup\{x\}}$ satisfies GMC above $y$ and BMC below $y$ for each $y \in Y$.

(b) For each finite set $Z$ with all outcomes ranked below $x$ the preference $\succcurlyeq$

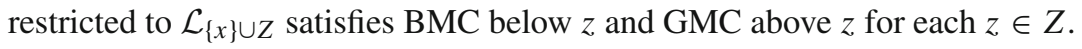

Before presenting the next result, we point out that, for obtaining extended PTfoundations for $\succcurlyeq$ on $\mathcal{L}$, we employ similar tools as in the derivation of Theorem 1 but restricted to each set $\mathcal{L}_{Y}$ for finite $Y \subset X$. In the presence of weak order, continuity and dominance, CSTP characterizes a general representation that is additive on each set $\mathcal{L}_{Y}$ for finite $Y \subset X$. Similar results were provided in Chew and Wakker (1996) for the general setup with ambiguity. When combined with the other properties, e-RMC implies extended PT on each set $\mathcal{L}_{Y}$, but it need not imply the existence of a uniquely determined reference point because the subset $Y$ may not contain the reference point. In general, the lack of structural assumptions on the set of outcomes $X$ means that a reference point $r \in X$ may not exist, for instance, as it is not part of the considered outcome set, even though preferences are sign-dependent. The following example illustrates. 
Example 4 Assume that $X=[-50,0) \cup(0,100]$ and that the preference $\succcurlyeq$ on $\mathcal{L}_{X}$ is represented by

$$
\begin{gathered}
V\left(p_{1}: x_{1}, \ldots, p_{n}: x_{n}\right)=\sum_{j=1}^{k-1} w^{+}\left(p_{j}^{d}\right)\left[u\left(x_{j}\right)-u\left(x_{j+1}\right)\right]+w^{+}\left(p_{k}^{d}\right) u\left(x_{k}\right) \\
\quad+\hat{w}^{-}\left(p_{k+1}^{c}\right) u\left(x_{k+1}\right) \\
\quad+\sum_{j=k+2}^{n} \hat{w}^{-}\left(p_{j}^{c}\right)\left[u\left(x_{j}\right)-u\left(x_{j-1}\right)\right],
\end{gathered}
$$

with strictly increasing and continuous weighting functions $w^{+}$and $w^{-}$, and a utility function defined as $u(x)=x$ if $x \in(0,100]$ and $u(x)=2 x$ if $x \in[-50,0)$, and $k$ denoting the number of positive outcomes in the prospect $\left(p_{1}: x_{1}, \ldots, p_{n}: x_{n}\right)$.

The preference in Example 4 satisfies all properties required for the existence of a general additive representation (weak order, Jensen-continuity, dominance, CSTP) and also e-RMC. However, the value 0 , which acts as a reference point, is not an outcome that is contained in the set $X$. Hence, the preference is sign-dependent, but no reference point within $X$ exists; the reference point is "outside the model."

Demanding convexity for the outcome set can circumvent the problems alluded to in Example 4. But even if the set of outcomes is a closed interval, we may have signdependent preferences but no reference point. This can be inferred from the following example that resembles features from Example 3.

Example 5 Assume that $X=[0,100]$ and that the preference $\succcurlyeq$ on $\mathcal{L}_{X} \backslash\{0\}$ is represented by

$$
\begin{aligned}
V\left(p_{1}: x_{1}, \ldots, p_{n-1}: x_{n-1}, p_{n}: 0\right)= & \sum_{j=1}^{n-2} w^{+}\left(p_{j}^{d}\right)\left[u\left(x_{j}\right)-u\left(x_{j+1}\right)\right] \\
& +w^{+}\left(p_{n-1}^{d}\right) u\left(x_{n-1}\right)-\left[\frac{p_{n}}{1-p_{n}}\right],
\end{aligned}
$$

with strictly increasing and continuous weighting functions $w^{+}$and $w^{-}$, and a utility function defined as $u(x)=x$ if $x \in(0,100]$ (that can discontinuously be extended to $u(0)=-1)$, and $n-1$ referring to the number of positive outcomes in the prospect evaluated by $V$.

To exclude preferences that are sign-dependent, but where a reference point cannot be identified, such as the preferences in Examples 4 and 5 one needs to add further structural assumptions for the set of outcomes or exclude extreme outcomes (e.g., requiring that $X$ is an open interval in $\mathbb{R}$ ). Alternatively, one can consider preferences where the reference point is outside the model as in the extended PT of Example 5 with an "imaginary" reference point $r$ *. We provide foundations for both cases. The first result provides a preference foundation for PT for the case that the set of outcomes is 
an open interval of the real numbers. As the set of outcomes is a connected separable topological space that does not contain a best or worst outcome, the reference point can be identified within the model.

Theorem 3 Assume $X=(a, b)$ for some real numbers $a<b$ and that the preference over outcomes agrees with the natural ordering of real numbers. The following two statements are equivalent for a preference relation $\succcurlyeq$ on $\mathcal{L}$ :

(i) The preference relation $\succcurlyeq$ on $\mathcal{L}$ is represented by $P T$.

(ii) The preference relation $\succcurlyeq$ is a Jensen-continuous weak order that satisfies dominance, the comonotonic sure thing principle for risk and extended reference point revealing midpoint consistency.

The probability weighting functions are uniquely determined. Further, if $w^{+}=w^{-}$, utility is cardinal as preferences agree with rank-dependent utility. Otherwise, the utility function is a ratio scale and the reference point $r \in X$ is unique.

The proof of Theorem 3 is in the Appendix. From that proof one can infer that Theorem 3 also applies for the case that $X=\mathbb{R}$, which is the most frequent assumption considered in the literature. The next result applies to the most general case in which no best and worst outcomes are allowed.

Theorem 4 Suppose $X$ is a set of outcomes that contains no extreme outcomes (i.e., $X$ has no best and no worst outcome). The following two statements are equivalent for a preference relation $\succcurlyeq$ on $\mathcal{L}$ :

(i) The preference relation $\succcurlyeq$ on $\mathcal{L}$ is represented by $P T$ with a possibly imaginary reference point.

(ii) The preference relation $\succcurlyeq$ is a Jensen-continuous weak order that satisfies dominance, the comonotonic sure thing principle for risk and extended reference point revealing midpoint consistency.

The probability weighting functions are uniquely determined. Further, if $w^{+}=w^{-}$, utility is cardinal as preferences agree with rank-dependent utility. Otherwise, the utility function is a ratio scale and, if the reference point $r \in X$, it is unique.

The proof of Theorem 4 is in the Appendix. Having explored how the probability midpoint tool can be used to obtain information about sign-dependence and reference points from a foundational perspective, we proceed to a practical application of midpoint consistency. The next subsection indicates how the midpoint tool can be applied to empirically test for sign-dependence.

\subsection{Detecting reference points empirically}

Our theoretical results, in particular the application of GMC and BMC as combined in RMC suggests that it is possible to test for sign-dependence using probability midpoints. Here we present a tool that can be used to experimentally implement such a test. Suppose, for simplicity of exposition, that we have the best outcome, labeled $G \in X$ (which may objectively be seen as a gain), and the worst outcome, labeled 
$L \in X$ (potentially regarded as a loss). Let there be finitely many outcomes ranked between $G$ and $L$, say $G \succ y_{1} \succ \cdots \succ y_{s} \succ L$ for some $s \geq 2$. Assume that this list of intermediate outcomes is exhaustive and that we have PT-preferences, but do not know if one of the $y_{j}$ 's is the reference point. An example of a procedure to identify the reference point involves repeated elicitations of probability midpoints and subsequent checks for consistency for those midpoints. This results in an algorithm searching for a reference point, as follows.

STEP 1: Fix a small probability $\alpha$ (e.g., $\alpha=0.15$ ) and probabilities $p, q$ (e.g., $p=0.1, q=0.2)$. Next consider the prospect $P_{y_{1}}^{\alpha}=\left(\alpha: G, 1-p-\alpha: y_{1}, p: L\right)$ and elicit the probability $\beta$ that makes a subject indifferent between $P_{y_{1}}^{\alpha}$ and $Q_{y_{1}}^{\beta}=$ ( $\left.\beta: G, 1-q-\beta: y_{1}, q: L\right)$. Such elicitations can be facilitated by using, for instance, choice lists in which outcomes are fixed and probabilities vary, as suggested in Holt and Laury (2002).

STEP 2: Replace $\alpha$ by $\beta$ in $P_{y_{1}}^{\alpha}$, hence obtaining $P_{y_{1}}^{\beta}$, and elicit $\gamma$ such that $P_{y_{1}}^{\beta} \sim$ $Q_{y_{1}}^{\gamma}$. This way the experimenter obtains a probability midpoint $\beta$ between $\alpha$ and $\gamma$.

STEP 3: Next, replace $y_{1}$ by $y_{2}$ in $P_{y_{1}}^{\alpha}$ and obtain the prospect $P_{y_{2}}^{\alpha}=(\alpha: G, 1-p-$ $\left.\alpha: y_{2}, p: L\right)$. Subsequently, elicit the probability $\tilde{q}$ that makes a subject indifferent between $P_{y_{2}}^{\alpha}$ and $Q_{y_{2}}^{\beta}=\left(\beta: G, 1-\tilde{q}-\beta: y_{2}, \tilde{q}: L\right)$.

STEP 4: As in Step 2, replace $\alpha$ by $\beta$ in $P_{y_{2}}^{\alpha}$, giving $P_{y_{2}}^{\beta}$, and elicit $\gamma^{*}$ such that $P_{y_{2}}^{\beta} \sim Q_{y_{2}}^{\gamma^{*}}$. The experimenter obtains a probability midpoint $\beta$ between $\alpha$ and $\gamma^{*}$. If $\gamma=\gamma^{*}$ we have observed a consistency, from which we conclude that $y_{1}$ and $y_{2}$ are outcomes with utilities of the same sign; we proceed to the next step. Otherwise, if $\gamma \neq \gamma^{*}$ we have an inconsistency, which can occur only if $y_{1}$ and $y_{2}$ have utilities of a different sign. Therefore, $y_{1}$ is identified as the reference point and the "search algorithm" stops.

STEP $(3+m)(m=2, \ldots, s)$ : If $\gamma=\gamma^{*}$ at Step $(2+m)$, repeat Steps $(1+m)$ and $(2+m)$, with $y_{m+1}$ and $y_{m}$ replacing $y_{m}$ and $y_{m-1}$, respectively.

If this procedure terminates after Step $\left(3+m^{*}\right)$ for some index $m^{*} \leq s$, we conclude that $y_{m} *$ is the reference point; otherwise, there is no reference point that affects the treatment of probabilities.

A few comments on the above procedure are in order. Obviously, there are alternative ways of implementing the above search procedure. For instance, one can start the procedure in Steps 1 and 2 at any outcome $y_{m}, m \in\{1, \ldots, s\}$ and adjust the subsequent elicitation steps, or one could elicit midpoints using outcome $y_{s}$ in Steps 3 and 4 in the above procedure and then continue the elicitation of midpoints by alternation between the remaining best and worst ranked outcomes that have not yet been identified as gains or losses by the procedure. If $X$ is an open interval in $\mathbb{R}$, one can use this procedure to narrow down the interval of outcomes in which the reference point is located by repeating the procedure on specific finite subsets $Y$ of $X$.

While the above search procedure appears compelling and, from a theoretically perspective, feasible, implementing the procedure in experiments would need to account for some practicalities. First, the probability interval is narrow such that small changes in stimuli in the form of actual probabilities may hardly be noticed by subjects. This can be circumvented by "scaling up" the stimuli, e.g., by framing choices as events 
resulting from draws using urns containing 100 or even 1000 equally likely balls. Second, the midpoint procedure is based on eliciting indifferences. Irrespective of whether indifferences are elicited by varying outcomes or by varying probabilities, such elicitation tasks are cognitively demanding for many subjects and appropriate experimental procedures are needed. The aforementioned choice lists design (Holt and Laury 2002), which invokes an final interpolation step, has proven to be quite an efficient mechanism in dealing with the issue of eliciting indifferences.

Third, the decision criterion in how far to tolerate differences between $\gamma$ and $\gamma^{*}$ in Step 4 of the above elicitation procedure usually needs to be specified as a rule where small differences can be regarded as a measurement error and large ones as a genuine inconsistency. What determines such bounds is essentially an empirical question, and setting appropriate thresholds can be based on existing data regarding empirically observed probability weighting functions. Fourth, chained measurements, as employed in our search procedure, have been criticized on the grounds of incentive compatibility and error propagation. Both aspects of the elicitation procedure are theoretically important, but empirically, these issues are not a serious concern as subjects treat choice tasks in isolation (Kahneman and Tversky 1979; Cubitt et al. 1998; see also Abdellaoui et al. 2005, on negligible error propagation).

Finally, in contrast to EU- or RDU-preferences, the choice of outcome stimuli to detect sign-dependence is important. The experimenter needs to ensure that the range of outcomes chosen to implement the above procedure is not too narrow (such that reference points are excluded) and likewise that the number of stimuli is not too large as this raises the number of required elicitations. This calls for a trade-off between precision of the method and the cognitive demands put on subjects that, in turn, can influence the precision in the obtained data. Finding the right balance is, however, a common challenge for all experimental studies.

\section{Discussion}

Here, we review some of the literature on endogenous reference points before we comment on the relation of our midpoint principle to midpoint notions developed elsewhere.

\subsection{Models with reference points}

The majority of existing PT-derivations assume the reference point is exogenously given (e.g., Tversky and Kahneman 1992; Wakker and Tversky 1993; Chateauneuf and Wakker 1999; Köbberling and Wakker 2003; Neilson 2006). In alternative models, attention has been paid to endogenous reference points that are choice-dependent (e.g., Gul 1991; Sugden 2003; Delquié and Cillo 2006; Bleichrodt 2007; Schmidt et al. 2008). Such multiple reference points are explicitly allowed for in the referencedependent theories of Munro and Sugden (2003) and Sagi (2006) where, motivated by the status quo effect, adjustments of preferences to new reference points are permitted. There the decision maker can be seen as having multiple preferences, each depending on a reference point. Those preferences are required to be consistent in the sense that 
they do not generate cyclical choices. Such consistency requirements for behavior are also appearing in theories that build on the classical revealed preference approach, however, by using choice functions that are reference-dependent, such as in Apesteguia and Ballester (2009) and Bossert and Sprumont (2009).

Multiple reference points can also be found in the choice model of Ok et al. (2015). Those reference points are feasible alternatives in a choice set, but they are always dominated by some other alternatives and, hence, are never revealed preferred. By contrast, the endogenous reference points in Shalev (2000, 2002), Köszegi and Rabin (2006, 2007), and Kőszegi (2010), correspond to a person's rational expectations held in the recent past, which in turn are determined in the so-called personal equilibrium. Since there may be multiple equilibria, the decision maker is required to choose the most preferred one, e.g., a preferred personal equilibrium in Kőszegi and Rabin (2006) and Köszegi (2010). Beyond the lack of uniqueness, the choice aspect is markedly different to the reference point concept in PT where, as pointed out by Shleifer (2012, p.1086), the reference point cannot be chosen deliberately.

Schmidt and Zank (2012) provide an alternative way to identify reference points from primitives by exploiting PT features, such as diminishing sensitivity of the utility (convexity for losses and concavity for gains) and consistent utility measurement paired with sign-dependence. The present approach is complementary to Schmidt and Zank (2012), and, unlike theirs, it does not impose structural richness on the set of outcomes. As a result, the present foundations for PT can be extended to more general settings like health and insurance where outcomes might be discrete, thus allowing for wider applications of PT.

\subsection{Outcome and probability midpoints}

Consistency requirements for outcomes are familiar in economics and finance and are commonly used for utility measurements or for comparative analyses. Specifically, the shape of utility functions can be inferred from preference-based outcome midpoints (Baillon et al. 2012, Theorem 2.2), where a strictly concave utility requires that the utility midpoint of two outcomes is always below the corresponding algebraic midpoint and, further, independent of the probabilities of those outcomes. Similarly, consistently lower midpoints indicate more concavity of one utility relative to the other. The utility midpoint tool, which applies likewise to expected utility and nonexpected utility theories, has been advanced further in Baillon et al. (2012) to compare the utility for risk with that for ambiguity.

Similarly to the comparison of utility functions based on outcome midpoints, attitudes toward probabilities can be inferred from comparisons of the probability weighting functions for risk and ambiguity (Abdellaoui et al. 2011) by adopting an analogous midpoint technique for probabilities. In Kuilen and Wakker (2011) it was demonstrated that probability midpoints are suitable for experimental studies and their elicitation is relatively easy. In empirical research, however, utility measurements were required prior to the elicitation of probability weighting functions (Abdellaoui 2000; Bleichrodt and Pinto 2000, and Kuilen and Wakker 2011). Our elicitation method and the derived results suggest that one can completely dispense of utility-based mea- 
surements when employing the probability midpoint tool. Indeed, we advance the probability midpoint tool in three different ways: Our PT-foundation delivers in one stroke a new preference tool for experimental testing and empirical measurements, a tool for comparative analyses and, more fundamentally, a method to identify reference points endogenously from behavior.

\section{Conclusion}

A consequence of reference-dependence is that risk behavior in PT is manifested through a combination of attitudes toward gains and losses, captured by a utility function (e.g., concave for gains and convex for losses), and attitudes toward probabilities of gains and losses, captured by corresponding probability weighting functions (e.g., inverse-S-shaped), which together imply a fourfold pattern of risk attitudes (Tversky and Kahneman 1992; Wu and Gonzalez 1996; Prelec 1998). ${ }^{12}$ Loss aversion, the component of risk attitude expressed as a stronger sensitivity toward losses as compared to equally sized gains (Wakker and Tversky 1993; Neilson 2002; Köbberling and Wakker 2005; Blavatskyy 2011) is a further cornerstone of PT that completes the picture on empirically observed choice behavior under risk. Yet, without knowledge of the location of the reference point, both loss aversion and the fourfold pattern become ambiguous concepts. While a model with an unspecified reference point gives additional flexibility for the analyst (e.g., it leads to an easier organization of experimental and field data, or it allows for framing effects to be incorporated), the absence of behavioral conditions that imply the existence of the reference point renders PT too general and it makes the model difficult to falsify. This is unwarranted both empirically and foundationally.

The importance of having sound preference foundations for decision models, in particular for PT, has recently been reiterated by Kothiyal et al. (2011, pp. 196-197). If a continuous utility is not available because outcomes are discrete (e.g., as in health or insurance), the relationship between the empirical primitive (i.e., the preference relation) and the assumptions of PT becomes unclear. In that case, one can no longer be sure that the PT-predictions are in line with the behavior underlying the preferences.

Our aim was to make PT falsifiable. We have addressed the foundational aspect of the reference point in PT under risk and showed that a specific consistency property for probability midpoints can be formulated in a way that allows for the identification of the reference point and, jointly, of PT. The conditions presented here are necessary and sufficient for PT; thus, they clarify which assumptions one makes by invoking the model. In particular, the new foundations highlight the difference between expected utility, rank-dependent utility and prospect theory in a transparent way. Before, this was not possible as foundations for PT in the von Neumann and Morgenstern setting were not available. In the presence of standard preference conditions, the two principles of good-news and bad-news midpoint consistency, when combined as in our RMC properties, are sufficient to obtain either RDU, a special case of PT, or PT with sign-

12 See also Wakker (2010), Abdellaoui et al. (2011), and the references therein for ambiguity. 
dependent probability weighting and a reference point endogenously revealed from behavior. This way, we have obtained a complete foundation for prospect theory.

Open Access This article is distributed under the terms of the Creative Commons Attribution 4.0 International License (http://creativecommons.org/licenses/by/4.0/), which permits unrestricted use, distribution, and reproduction in any medium, provided you give appropriate credit to the original author(s) and the source, provide a link to the Creative Commons license, and indicate if changes were made.

\section{Appendix}

Before presenting the proofs of the lemmas and the theorems, we provide further details on Example 1 as it clarifies the nature of the additive representation when $X$ consists of exactly three strictly rank-ordered outcomes.

\section{Three strictly ordered outcomes}

FURTHER ELABORATION ON EXAMPLE 1: Recall the additive representation $V\left(p_{1}\right.$ : $\left.x_{1}, p_{2}: x_{2}, p_{3}: x_{3}\right)=V_{1}\left(p_{1}\right)+V_{2}\left(p_{1}+p_{2}\right)$ in Example 1. For all probabilities $p \in[0,1]$, set

$$
w^{+}(p)=\frac{V_{1}(p)-V_{1}(0)}{V_{1}(1)-V_{1}(0)},
$$

which is well defined as $V_{1}$ is bounded at 1 . Thus defined, $w^{+}$is a probability weighting function. Set $u\left(x_{1}\right):=V_{1}(1)-V_{1}(0)>0$, and obtain that

$$
V_{1}\left(p_{1}\right)=w^{+}\left(p_{1}\right) u\left(x_{1}\right)+V_{1}(0)
$$

Next, define

$$
w^{-}(p)=\frac{V_{2}(p)-V_{2}(0)}{V_{2}(1)-V_{2}(0)} .
$$

We set $u\left(x_{3}\right)=V_{2}(0)-V_{2}(1)<0$ and obtain

$$
\begin{aligned}
V_{2}\left(p_{1}+p_{2}\right) & =w^{-}\left(p_{1}+p_{2}\right)\left[V_{2}(1)-V_{2}(0)\right]+V_{2}(0) \\
& =-w^{-}\left(p_{1}+p_{2}\right) u\left(x_{3}\right)+V_{2}(0)+V_{2}(1)-V_{2}(1) \\
& =\left[1-w^{-}\left(p_{1}+p_{2}\right)\right] u\left(x_{3}\right)+V_{2}(1) \\
& =\hat{w}^{-}\left(p_{3}\right) u\left(x_{3}\right)+V_{2}(1) .
\end{aligned}
$$

To obtain the last equation, we have used the dual of $w^{-}$and the fact that $p_{1}+p_{2}=$ $1-p_{3}$. 
Continuity of the weighting functions implies that

$$
\begin{aligned}
\lim _{\substack{p+q \rightarrow 0 \\
0<p+q<1}} V(p, 1-p-q, q) & =\lim _{\substack{p+q \rightarrow 0 \\
0<p+q<1}}\left[w^{+}(p) u\left(x_{1}\right)+V_{1}(0)+\hat{w}^{-}(q) u\left(x_{3}\right)+V_{2}(1)\right] \\
& =V_{1}(0)+V_{2}(1) .
\end{aligned}
$$

To see that the just obtained sum is immaterial for the preference between prospects, we note that

$$
\begin{aligned}
P \succcurlyeq Q \Leftrightarrow & V_{1}\left(p_{1}\right)+V_{2}\left(p_{1}+p_{2}\right) \geq V_{1}\left(q_{1}\right)+V_{2}\left(q_{1}+q_{2}\right) \\
\Leftrightarrow & w^{+}\left(p_{1}\right) u\left(x_{1}\right)+V_{1}(0)+\hat{w}^{-}\left(p_{3}\right) u\left(x_{3}\right)+V_{2}(1) \geq w^{+}\left(q_{1}\right) u\left(x_{1}\right) \\
& \quad+V_{1}(0)+\hat{w}^{-}\left(q_{3}\right) u\left(x_{3}\right)+V_{2}(1) \\
\Leftrightarrow & w^{+}\left(p_{1}\right) u\left(x_{1}\right)+\hat{w}^{-}\left(p_{3}\right) u\left(x_{3}\right) \geq w^{+}\left(q_{1}\right) u\left(x_{1}\right)+\hat{w}^{-}\left(q_{3}\right) u\left(x_{3}\right) .
\end{aligned}
$$

By continuity in probabilities, $p \in(0,1)$ exists, such that $\left(1: x_{2}\right) \sim\left(p: x_{1}, 1-\right.$ $\left.p: x_{3}\right)$. This implies $0=w^{+}(p) u\left(x_{1}\right)+\hat{w}^{-}(1-p) u\left(x_{3}\right)$. We must, therefore, set $u\left(x_{2}\right)=0$. We have obtained a PT-representation from the additive representation of the preference relation and have identified the reference point $r:=x_{2}$.

Recall that uniqueness results for additive representations specify joint cardinality of $V_{1}$ and $V_{2}$. Thus, if instead of the latter pair we start our derivation of weighting functions and utility with $A V_{1}+B_{1}$ and $A V_{2}+B_{2}$ for some positive $A$ and real $B_{1}$ and $B_{2}$, we obtain $w^{+}$and $\hat{w}^{-}$as above, showing that the weighting functions are uniquely determined. If $w^{+} \neq w^{-}$the constraint $u\left(x_{2}\right)=0$ is binding for all admissible utility functions, such that for a different utility, $\tilde{u}$, we obtain $\tilde{u}\left(x_{j}\right)=A u\left(x_{j}\right)$; thus, utility is a ratio scale. It can be shown that, if $w^{-}$is identical to $w^{+}$, this PT-representation is in fact an RDU-representation to which the corresponding uniqueness results apply. Hence, PT holds for the case that $X$ contains exactly three strictly ranked outcomes and the preference is represented by a bounded additively separable function.

Example 1 and the just provided elaborations show that, for the case of exactly three strictly ordered outcomes, no additional preference requirements are needed to derive PT, provided that we have an additive representation with bounded functions. If exactly one of those functions is unbounded, then extended PT holds trivially, while in the case when both functions are unbounded, we can only identify $r=x_{2}$ as the reference point, but cannot identify the probability weighting functions separately of utility. Wakker (1993, Theorem 3.2) provides conditions under which an additive representation can be obtained for the preference restricted to the set of all prospects except the extreme ones, i.e., for $\succcurlyeq$ on $\mathcal{L} \backslash\left\{x_{1}, x_{3}\right\}$. Wakker (1993, Proposition 3.5) indicates that, if the additive representation consists of proportional functions, they can be assumed to be finite at extreme outcomes; then the additive functions are bounded. Invoking such proportionality requirements comes down to establishing RDU (see Abdellaoui 2002), i.e., PT without sign-dependence. Thus, to allow for sign-dependence while excluding unboundedness of the functions $V_{1}$ and $V_{2}$, weaker conditions than proportionality are warranted. For instance, one can require conditions, which can also be formulated for the case of more than three strictly ordered outcomes, such as those in Wakker (1993, Theorem 3.3(c)), which ensure that the functions in the additive representation 
are bounded. Given our general approach, we have allowed for unbounded additive representations and have provided behavioral conditions under which extended PT holds.

Having elaborated on the case when the set of outcomes contains exactly three strictly ordered outcomes, we assume, henceforth, that $X$ contains at least four strictly ordered outcomes. For this case Lemma 1 gives a preference foundation for an additive representation of the preference $\succcurlyeq$ on $\mathcal{L} \backslash\left\{x_{1}, x_{n}\right\}$. The proof of the lemma follows from Wakker (1993, Theorem 3.2); see also Zank (2010).

\section{Proofs}

ProOF OF LEMMA 2: If RDU holds for $\succcurlyeq$ on $\mathcal{L}$, then GMC above $x$ follows for all outcomes $x \in X$, as shown in Zank (2010). Thus, for the case when there are no gains or the case when there are no losses, the statement in the lemma holds. Next we assume that we have sign-dependence and that PT holds for $\succcurlyeq$ on $\mathcal{L}$ or that extended PT holds on $\mathcal{L} \backslash\left\{x_{n}\right\}$. Then, $x_{1}$ is a gain and $x_{n}$ is a loss. Let $x_{m}, m \in\{2, \ldots, n-1\}$, be a gain or the reference point. Suppose that $P=\left(\alpha: x_{1}, p_{m}-\alpha: x_{m}, p_{m+1}: x_{m+1}, \ldots, p_{n}: x_{n}\right)$ and $Q=\left(\beta: x_{1}, q_{m}-\beta: x_{m}, q_{m+1}: x_{m+1}, \ldots, q_{n}: x_{n}\right)$ and that the indifferences

$$
P \sim Q \text { and }(\beta-\alpha)_{m, 1} P \sim(\gamma-\beta)_{m, 1} Q
$$

hold for $\alpha<\beta<\gamma$ such that $P, Q,(\beta-\alpha)_{m, 1} P$, and $(\gamma-\beta)_{m, 1} Q$ are well defined (the case $\alpha=\beta$ which implies $\beta=\gamma$ is trivial). Let $\tilde{m} \in\{1, \ldots, m\}$ be arbitrary. We show that $(\beta-\alpha)_{m, \tilde{m}} P \sim(\gamma-\beta)_{m, \tilde{m}} Q$ must hold.

Substitution of (extended) PT into $P \sim Q$ and $(\beta-\alpha)_{m, 1} P \sim(\gamma-\beta)_{m, 1} Q$, taking differences of the resulting equation and cancelling common terms, gives: $w^{+}(\beta)-w^{+}(\alpha)=w^{+}(\gamma)-w^{+}(\beta)$, i.e., $\beta$ is a $w^{+}$-midpoint between $\alpha$ and $\gamma$, or equivalently,

$$
w^{+}(\beta)=\frac{w^{+}(\gamma)+w^{+}(\alpha)}{2} .
$$

Assume, to the contrary, that $(\beta-\alpha)_{m, \tilde{m}} P \succ(\gamma-\beta)_{m, \tilde{m}} Q$. Substitution of (extended) PT into $P \sim Q$ and $(\beta-\alpha)_{m, \tilde{m}} P \succ(\gamma-\beta)_{m, \tilde{m}} Q$, taking differences between the resulting equation and, respectively, inequality, implies

$$
w^{+}(\beta)<\frac{w^{+}(\gamma)+w^{+}(\alpha)}{2},
$$

contradicting the preceding equation. The case $(\beta-\alpha)_{m, \tilde{m}} P \prec(\gamma-\beta)_{m, \tilde{m}} Q$ leads to a similar contradiction; hence, $(\beta-\alpha)_{m, \tilde{m}} P \sim(\gamma-\beta)_{m, \tilde{m}} Q$ must hold. As $\tilde{m} \in\{1, \ldots, m\}$ was arbitrary, it follows that $(\beta-\alpha)_{m, \tilde{m}} P \sim(\gamma-\beta)_{m, \tilde{m}} Q$ holds for all $\tilde{m} \in\{1, \ldots, m\}$. Hence, GMC above $x_{m}$ holds. As $m \in\{2, \ldots, n-1\}$ was arbitrarily chosen such that $x_{m}$ is not a loss, this completes the proof of Lemma 2. 
Proof of Lemma 3: This proof is similar to the proof of Lemma 2.

Proof of Theorem 1: The derivation of statement (ii) from statement (i) follows from the fact that extended PT is an additive representation of the preference $\succcurlyeq$ on $\mathcal{L} \backslash\left\{x_{1}\right\}$, or on $\mathcal{L} \backslash\left\{x_{n}\right\}$, or on $\mathcal{L}$. Then statement (ii) of Lemma 1 applies. RMC follows from Lemmas 2 and 3. This completes the derivation of statement (ii).

We now prove that statement (ii) implies statement (i) of the theorem. We note that statement (ii) of the theorem implies statement (ii) of Lemma 1. Hence, we can assume that the preference $\succcurlyeq$ on $\mathcal{L} \backslash\left\{x_{1}, x_{n}\right\}$ is represented by an additive function

$$
V(P)=\sum_{j=1}^{n-1} V_{j}\left(p_{j}^{d}\right),
$$

with continuous strictly increasing functions $V_{1}, \ldots, V_{n-1}:[0,1] \rightarrow \mathbb{R}$ which are bounded except $V_{1}$ (which can be unbounded at $p=1$ ) and $V_{n-1}$ (which can be unbounded at $p=0$ ).

Next we invoke RMC. It says that for each $m \in\{2, \ldots, n-1\}$ the preference satisfies one of the following three conditions: (I) GMC above outcome $x_{m}$, or (II) BMC below outcome $x_{m-1}$, or (III) it jointly satisfies both GMC above $x_{m}$ and BMC below $x_{m-1}$. We observe that GMC above $x_{2}$ holds trivially (and similarly BMC below $x_{n-1}$ holds trivially). Next we consider several cases.

CASE 1: Suppose that GMC above $x_{3}$ does not hold. Then, RMC implies that BMC holds below $x_{2}$. Using similar arguments as in Zank (2010), one obtains by induction, first locally then globally, that the functions $V_{2}, \ldots, V_{n-1}$ are proportional on $(0,1)$. Following Wakker (1993, Proposition 3.5) these functions are bounded and can continuously be extended to all of $[0,1]$. Define

$$
w^{-}(p):=\frac{V_{2}(p)-V_{2}(0)}{V_{2}(1)-V_{2}(0)}
$$

for all probabilities $p \in[0,1]$. This is a well-defined (continuous and strictly increasing) probability weighting function. Further, by proportionality of the functions $V_{2}, \ldots, V_{n-1}$ it follows that for all $j=2, \ldots, n-1$ we have

$$
w^{-}(p)=\frac{V_{j}(p)-V_{j}(0)}{V_{j}(1)-V_{j}(0)}
$$

for all probabilities $p \in[0,1]$. From the latter expression we obtain

$$
V_{j}(p)=w^{-}(p)\left[V_{j}(1)-V_{j}(0)\right]+V_{j}(0)
$$

for all $j=2, \ldots, n-1$, and for all probabilities $p \in[0,1]$.

As GMC does not hold above $x_{3}, V_{1}$ cannot be proportional to $V_{2}$ (or to the $V_{j}$ 's, $j=3, \ldots, n-1)$. This means that $V_{2}$-midpoints exist that are not $V_{1}$-midpoints. In particular, GMC above $x_{m}$ cannot hold if $m>2$. Therefore, $x_{2}$ is a reference point. Next we define $u\left(x_{2}\right)=0$ and $u\left(x_{j+1}\right)-u\left(x_{j}\right)=V_{j}(0)-V_{j}(1)<0$ for 
all $j=2, \ldots, n-1$. This way, we have obtained a utility function for all outcomes $x_{j}, j=2, \ldots, n$. Then, for all $j=2, \ldots, n-1$, and for all probabilities $p \in[0,1]$, we obtain

$$
\begin{aligned}
V_{j}(p) & =w^{-}(p)\left[u\left(x_{j}\right)-u\left(x_{j+1}\right)\right]+V_{j}(0) \\
& =w^{-}(p)\left[u\left(x_{j}\right)-u\left(x_{j+1}\right)\right]+V_{j}(0)-V_{j}(1)+V_{j}(1) \\
& =w^{-}(p)\left[u\left(x_{j}\right)-u\left(x_{j+1}\right)\right]+u\left(x_{j+1}\right)-u\left(x_{j}\right)+V_{j}(1) \\
& =\left[1-w^{-}(p)\right]\left[u\left(x_{j+1}\right)-u\left(x_{j}\right)\right]+V_{j}(1) .
\end{aligned}
$$

Substitution into the additive representation for the preference on $\mathcal{L} \backslash\left\{x_{1}\right\}$ implies that Eq. (9) can be written as

$$
V(P)=V_{1}\left(p_{1}\right)+\sum_{j=2}^{n-1}\left[1-w^{-}\left(p_{j}^{d}\right)\right]\left[u\left(x_{j+1}\right)-u\left(x_{j}\right)\right]+\sum_{j=2}^{n-1} V_{j}(1) .
$$

We observe that the last term in the representation is a constant that cancels out when comparing two arbitrary prospects; hence, it is meaningless for the preference and can be dropped. Further, by the definition of the dual weighting function, we have $\left[1-w^{-}\left(p_{j}^{d}\right)\right]=\hat{w}^{-}\left(p_{j+1}^{c}\right)$. Using these observations, our representation is rewritten as

$$
V(P)=V_{1}\left(p_{1}\right)+\sum_{j=2}^{n-1} \hat{w}^{-}\left(p_{j+1}^{c}\right)\left[u\left(x_{j+1}\right)-u\left(x_{j}\right)\right] .
$$

If $V_{1}$ is unbounded at 1 we cannot extend this representation to the degenerate prospect that gives $x_{1}$ for sure. For that case, we obtain an extended PT-representation for the preference on $\mathcal{L} \backslash\left\{x_{1}\right\}$. As the functions $V_{j}, j=1, \ldots, n$, were jointly cardinal, it follows that $w^{-}$is uniquely determined. Further, because of the requirement that $u\left(x_{2}\right)=0$ the utility function is a ratio scale. The outcome $x_{k}$ with $k=2$ is unique with the property that the functions $V_{j}, j \geq k$, have common probability midpoints that are not common midpoints for the functions $V_{j}, j<k$. Hence, the reference point $r:=x_{2}$ is unique.

If $V_{1}$ is bounded, we can continuously extend the function to all of $[0,1]$ and we obtain a representation on $\mathcal{L}$, thus including the extreme outcome $x_{1}$. Then we define

$$
w^{+}(p)=\frac{V_{1}(p)-V_{1}(0)}{V_{1}(1)-V_{1}(0)}
$$

and obtain

$$
V_{1}(p)=w^{+}(p)\left[V_{1}(1)-V_{1}(0)\right]+V_{1}(0)
$$


Further, we set $V_{1}(1)-V_{1}(0)=u\left(x_{1}\right)-u\left(x_{2}\right)>0$, such that our representation is given by

$$
V(P)=w^{+}\left(p_{1}\right)\left[u\left(x_{1}\right)-u\left(x_{2}\right)\right]+\sum_{j=2}^{n-1} \hat{w}^{-}\left(p_{j+1}^{c}\right)\left[u\left(x_{j+1}\right)-u\left(x_{j}\right)\right]
$$

where we have dropped the constant $V_{1}(0)$ as it commonly occurs in the evaluation of each prospect. Hence, PT represents $\succcurlyeq$ on $\mathcal{L}$. Uniqueness results for $w^{-}, u$, and $x_{2}$ follow by similar arguments as in the case where $V_{1}$ was unbounded. Further, by joint cardinality of the functions in the additive representation, the normalization used to define $w^{+}$implies that the latter is unique. This completes the proof for the case when GMC does not hold above $x_{3}$.

CASE 2: Next we consider the case when BMC does not hold below $x_{n-2}$. Then, RMC implies that GMC holds above $x_{n-1}$. Using similar arguments as in Zank (2010), one obtains by induction, first locally then globally, that the functions $V_{1}, \ldots, V_{n-2}$ are proportional on $(0,1)$. Following Wakker (1993, Proposition 3.5) these functions are bounded and can continuously be extended to all of $[0,1]$. We define

$$
w^{+}(p)=\frac{V_{1}(p)-V_{1}(0)}{V_{1}(1)-V_{1}(0)}
$$

for all probabilities $p \in[0,1]$. This is a well-defined (continuous and strictly increasing) probability weighting function. Further, by proportionality of the functions $V_{1}, \ldots, V_{n-2}$ it follows that for all $j=1, \ldots, n-2$ we have

$$
w^{+}(p)=\frac{V_{j}(p)-V_{j}(0)}{V_{j}(1)-V_{j}(0)}
$$

for all probabilities $p \in[0,1]$. From the latter expression we obtain

$$
V_{j}(p)=w^{+}(p)\left[V_{j}(1)-V_{j}(0)\right]+V_{j}(0)
$$

for all $j=1, \ldots, n-2$, and for all probabilities $p \in[0,1]$.

As BMC does not hold below $x_{n-2}, V_{n-1}$ cannot be proportional to $V_{n-2}$ (nor to any of the other $V_{j}$ 's, $\left.j=1, \ldots, n-3\right)$. This means that $V_{n-2}$-midpoints exist, which are not $V_{n-1}$-midpoints. In particular, BMC below $x_{m}$ cannot hold if $m<n-2$. Therefore, $r:=x_{n-1}$ is (the uniquely determined) reference point. We now use similar arguments to those in Case 1, first to define utility for the reference point $\left(u\left(x_{n-1}\right)=0\right)$ and, iteratively, for the gains $u\left(x_{j}\right)-u\left(x_{j+1}\right)=V_{j}(1)-V_{j}(0)$ for $j=1, \ldots, n-2$, and second to obtain the representation

$$
V(P)=\sum_{j=1}^{n-2} w^{+}\left(p_{j}^{d}\right)\left[u\left(x_{j}\right)-u\left(x_{j+1}\right)\right]+V_{n-1}\left(p_{n-1}^{d}\right),
$$


where constant terms that are irrelevant for comparing prospects have been dropped. If $V_{n-1}$ is unbounded at 0 then we have obtained extended PT for $\succcurlyeq$ on $\mathcal{L} \backslash\left\{x_{n}\right\}$. Uniqueness results for $w^{+}$and $u$ follow from the construction of the corresponding functions and the joint cardinality of functions in the additive representation. If $V_{n-1}$ is bounded at 0 we can continuously extend $V_{n-1}$ and obtain an additive representation with bounded functions for $\succcurlyeq$ on $\mathcal{L}$ including outcome $x_{n}$. Then we define

$$
w^{-}(p)=\frac{V_{n-1}(p)-V_{n-1}(0)}{V_{n-1}(1)-V_{n-1}(0)}
$$

for all probabilities $p \in[0,1]$. Setting $u\left(x_{n}\right)-u\left(x_{n-1}\right)=V_{n-1}(0)-V_{n-1}(1)<0$ and substituting into the above representation gives PT (after dropping the constant term $\left.V_{n-1}(0)\right)$ with the corresponding uniqueness results. This completes the proof for the case when BMC does not hold below $x_{n-2}$.

CASE 3: Assume that there is some index $\tilde{m} \in\{3, \ldots, n-2\}$ such that GMC above $x_{\tilde{m}}$ holds, but GMC above $x_{\tilde{m}+1}$ does not hold. This means that $X$ consists of at least five strictly rank-ordered outcomes. By RMC it follows that BMC below $x_{\tilde{m}}$ holds. Following a similar line of arguments as used for the derivations in Cases 1 and 2 above, we first find that $V_{\tilde{m}}, \ldots, V_{n-1}$, respectively, that $V_{1}, \ldots, V_{\tilde{m}-1}$ are proportional. Proportionality implies that these functions are bounded and our additive representation can be extended to $\mathcal{L}$. As GMC above $x_{\tilde{m}}$ holds but GMC above $x_{\tilde{m}+1}$ does not hold, the functions $V_{\tilde{m}-1}$ and $V_{\tilde{m}}$ cannot be proportional. This implies that BMC below $x_{\tilde{m}-1}$ cannot hold. Therefore, $x_{\tilde{m}}$ is unique with the property that $V_{j}$-midpoints agree for all $j \in\{1, \ldots, \tilde{m}-1\}$ and that $V_{j}$-midpoints agree for all $j \in\{\tilde{m}, \ldots, n-1\}$. Hence, $r:=x_{\tilde{m}}$ is the unique reference point. As in Cases 1 and 2 , we can now uniquely define weighting functions for probabilities of gains and losses, respectively, and a ratio-scale utility with $u\left(x_{\tilde{m}}\right)=0$. Hence, PT represents the preference on $\mathcal{L}$. This completes the proof of Case 3 .

CASE 4: The final case to consider is when two distinct outcomes $x_{m^{\prime}} \succ x_{m^{\prime \prime}}$ with $m^{\prime}, m^{\prime \prime} \in\{2, \ldots, n-1\}$ exist, such that GMC above $x_{m^{\prime \prime}}$ holds and also BMC below $x_{m^{\prime}-1}$ holds. Then, we obtain proportionality of $V_{1}, \ldots, V_{m^{\prime \prime}}$ and proportionality of $V_{m^{\prime}-1}, \ldots, V_{n-1}$. As $m^{\prime}<m^{\prime \prime}$ it follows that all functions in the additive representation are proportional. Using a similar line of argument as in Zank (2010), we obtain an RDU-representation for preference on $\mathcal{L}$ with the corresponding uniqueness results. This completes Case 4.

Cases 1-4 cover all possibilities. This completes the proof of Theorem 1.

Proof of Theorem 2: The proof that statement (i) implies statement (ii) is similar to the corresponding proof in Theorem 1. To prove statement (i) from statement (ii) we note that b-RMC implies RMC. Hence, PT or extended PT holds. Further, as GMC above $x_{3}$ holds and BMC below $x_{n-2}$ holds, Cases 1 and 2 in the proof of Theorem 1 do not apply. Therefore, only Cases 3 and 4 need to be dealt with; the proof is completely analogous to the proof of Theorem 1; hence, PT holds. This proves statement (i) from statement (ii). Uniqueness results also follow from Case 3, respectively, Case 4, of the proof of Theorem 1. This concludes the proof of Theorem 2. 
Proof of Theorem 3: The proof that statement (i) implies statement (ii) is standard. In particular, for each finite set of at least four strictly ordered outcomes, CSTP follows from Theorem 1 when considering the preference restricted to prospects over that set. Further, if PT holds for $\succcurlyeq$ on $\mathcal{L}$, then either we have sign independence or we have sign-dependence. In the former case RDU holds and e-RMC follows from Theorem 4 in Zank (2010). In the latter case it must be that RDU represents $\succcurlyeq$ restricted to $\mathcal{L}_{Y \cup\{r\}}$ whenever $Y$ consists of gains and RDU represents $\succcurlyeq$ restricted to $\mathcal{L}_{\{r\} \cup Z}$ whenever $Z$ consists of losses. That is, for all $x \succcurlyeq r$, statement (a) of e-RMC holds and for all $x \preccurlyeq r$ statement (b) of e-RMC holds. Hence, e-RMC holds.

Next we prove statement (i) from statement (ii). In what follows, we consider only subsets $Y$ of $X=(a, b)$ with at least four strictly ordered outcomes, since prospects that assign positive probability to fewer outcomes are contained in these sets, such that the derived representations apply to them too. First we show that the preference on $\mathcal{L}$ is represented by a general additive representation. That is, the preference is represented by a functional $V: \mathcal{L} \rightarrow \mathbb{R}$ that, for each nonempty set $Y=\left\{y_{1}, \ldots, y_{n}\right\}$ with $y_{1} \succ \cdots \succ y_{n}$, of outcomes, evaluates prospects from $\mathcal{L}_{Y}$ by

$$
V\left(p_{y_{1}}: y_{1}, \ldots, p_{y_{n}}: y_{n}\right)=\sum_{j=1}^{n-1} V_{j}^{Y}\left(p_{y_{j}}^{d}\right)
$$

as in Lemma 1, except that all functions $V_{j}^{Y}$ are bounded. $W$ is also a general additive representation of $\succcurlyeq$ on $\mathcal{L}$ if and only if for each nonempty set $Y=\left\{y_{1}, \ldots, y_{n}\right\}$ with $y_{1} \succ \cdots \succ y_{n}$, of outcomes, we have $W_{j}^{Y}=A V_{j}^{Y}+B_{j}$ for constants $A>0$ and $B_{j} \in \mathbb{R}$.

Assume an arbitrary subset $Y=\left\{y_{1}, \ldots, y_{n}\right\}$ with $y_{1} \succ \cdots \succ y_{n}$ for a natural number $n \geq 4$. We consider the restriction of the preference $\succcurlyeq$ on the set of prospects over outcomes in $Y$, denoted $\mathcal{L}_{Y}$. This restriction inherits from $\succcurlyeq$ on $\mathcal{L}$ all properties stated in statement (ii) of Lemma 1. Hence, the preference on $\mathcal{L}_{Y}$ has an additive representation $V=\sum_{j=1}^{n-1} V_{j}^{Y}$. As $Y$ was arbitrary, this conclusion holds for all finite subsets of $X$ that contain at least four strictly ordered outcomes.

Since the set of outcomes $X$ is open, a best or a worst outcome in $X$ does not exist; hence, we can always find outcomes $y_{0} \succ y_{1}$ and $y_{n} \succ y_{n+1}$ and consider the additive representation of the preference on $\mathcal{L}_{Y \cup\left\{y_{1}, y_{n+1}\right\}}$ denoted $\tilde{V}=\sum_{j=0}^{n} \tilde{V}_{j}^{\left\{y_{0}\right\} \cup Y \cup\left\{y_{n+1}\right\}}$. As both functions are representations of the preference on $\mathcal{L}_{Y}$, by the uniqueness results we can set $V_{j}^{Y}=\tilde{V}_{j}^{\left\{y_{0}\right\} \cup Y \cup\left\{y_{n+1}\right\}}$ for all $j=1, \ldots, n-1$. In particular, this shows that $V_{1}^{\left\{y_{0}\right\} \cup Y \cup\left\{y_{n+1}\right\}}$ and $V_{n-1}^{\left\{y_{0}\right\} \cup Y \cup\left\{y_{n+1}\right\}}$ are bounded. As before, given that $Y$ was chosen arbitrarily, this conclusion holds for all corresponding additive representations.

Let now $Y, Y^{\prime}$ be two arbitrary finite subsets of $X$ each containing at least four strictly ordered outcomes. Consider the corresponding additive representations $V^{Y}$ and $V^{Y^{\prime}}$ of the preference restricted to $\mathcal{L}_{Y}$ and $\mathcal{L}_{Y^{\prime}}$, respectively, and further the additive representation $V^{Y \cup Y^{\prime}}$ of the preference restricted to $\mathcal{L}_{Y \cup Y^{\prime}}$. As $\mathcal{L}_{Y} \subset \mathcal{L}_{Y \cup Y^{\prime}}$ and $\mathcal{L}_{Y^{\prime}} \subset \mathcal{L}_{Y \cup Y^{\prime}}$ the latter representation also represents the preference on $\mathcal{L}_{Y}$ and on $\mathcal{L}_{Y^{\prime}}$. Given the uniqueness results for additive representations, we can choose $V_{j}^{Y}=V_{j}^{Y \cup Y^{\prime}}$ for all $y_{j} \in Y$ and, similarly, $V_{j}^{Y^{\prime}}=V_{j}^{Y \cup Y^{\prime}}$ for all $y_{j} \in Y^{\prime}$. As $Y$ and 
$Y^{\prime}$ were chosen arbitrarily, we conclude that a general additive representation $V$ for $\succcurlyeq$ on $\mathcal{L}$ exists.

Next we invoke e-RMC to show that $V$ is a PT-functional. We consider four cases. CASE I: Suppose that for all $x \in X$ and sets $Z=\left\{z_{1}, \ldots, z_{n}\right\}$ with $x \succ z_{1} \succ \cdots \succ z_{n}$ for a natural number $n \geq 3$, BMC below $z_{j}$ holds for each $j=1, \ldots, n-1$ and that

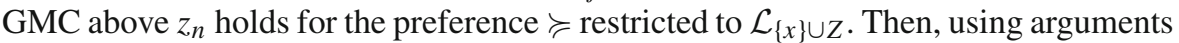

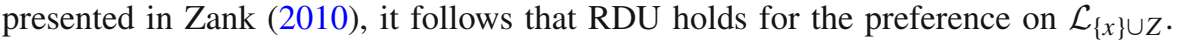
This implies that, for each set of outcomes $Z=\left\{z_{1}, \ldots, z_{n}\right\}$ with $z_{1} \succ \cdots \succ z_{n}$ for a natural number $n \geq 3$, RDU represents $\succcurlyeq$ on $\mathcal{L}_{Z}$. Hence, $V$ is an RDU-representation of $\succcurlyeq$ on $\mathcal{L}$. This is the special case of PT with sign independence. The uniqueness results for RDU-representations apply.

CASE II: Suppose that for all $x \in X$ and sets $Y=\left\{y_{1}, \ldots, y_{n}\right\}$ with $y_{1} \succ \cdots \succ y_{n} \succ x$ for $n \geq 3$, GMC above $x$ holds and BMC below $y_{j}$, for $j=2, \ldots, n$. Then, using arguments presented in Zank (2010), it follows that RDU holds for the preference on $\mathcal{L}_{Y \cup\{x\}}$. This implies that, for each set of outcomes $Y=\left\{y_{1}, \ldots, y_{n}\right\}$ with $y_{1} \succ \cdots \succ$ $y_{n}$ for $n \geq 3$, RDU represents $\succcurlyeq$ on $\mathcal{L}_{Y}$. Hence, $V$ is an RDU-representation to which the corresponding uniqueness results apply, i.e., $V$ is a PT-representation with sign independence, of $\succcurlyeq$ on $\mathcal{L}$.

CASE III: Suppose that for an outcome $x \in X$ and a set $Z=\left\{z_{1}, \ldots, z_{n}\right\}$ with

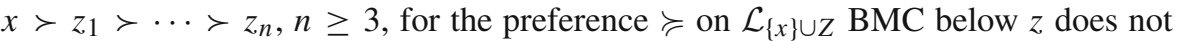
hold for an outcome $z \in Z$ or GMC above $z^{\prime}$ does not hold for an outcome $z^{\prime} \in Z$.

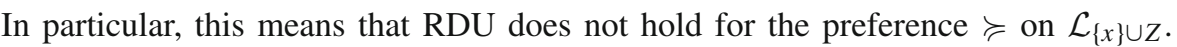
Then e-RMC implies that for all $Y=\left\{y_{1}, \ldots, y_{n^{\prime}}\right\}$ with $y_{1} \succ \cdots \succ y_{n^{\prime}} \succ x$, BMC below $y$ holds and GMC above $y$ holds for all $y \in Y$ for the preference $\succcurlyeq$ on $\mathcal{L}_{Y \cup\{x\}}$. By Theorem 4 in Zank (2010) this implies that RDU represents the preference $\succcurlyeq$ on $\mathcal{L}_{Y \cup\{x\}}$ for all $Y=\left\{y_{1}, \ldots, y_{n^{\prime}}\right\}$ with $y_{1} \succ \cdots \succ y_{n^{\prime}} \succ x$. That is, RDU holds for $\succcurlyeq$ on $\mathcal{L}_{\{y \in X \mid y \succcurlyeq x\}}$. Given our assumption that for the set $Z=\left\{z_{1}, \ldots, z_{n}\right\}$ and the

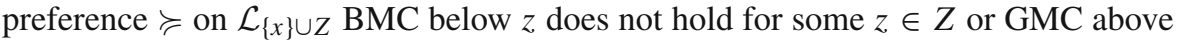
$z^{\prime}$ does not hold for some $z^{\prime} \in Z$, it cannot be that for all $x^{\prime} \in X$ RDU holds for $\succcurlyeq$ on $\mathcal{L}_{\left\{y \in X \mid y \succcurlyeq x^{\prime}\right\}}$ (for, otherwise, by Case II, RDU holds for $\succcurlyeq$ on $\mathcal{L}$ and in particular for

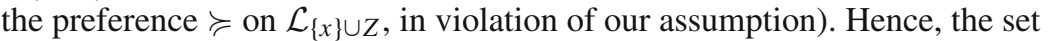

$$
X^{\prime}:=\left\{x^{\prime} \in X \mid \text { RDU holds for } \succcurlyeq \text { on } \mathcal{L}_{\left\{y \in X \mid y \succcurlyeq x^{\prime}\right\}}\right\}
$$

is bounded from below. Let $x_{*}$ be the outcome such that RDU holds for $\succcurlyeq$ on $\mathcal{L}_{\left\{y \in X \mid y \succ x_{*}\right\}}$ and no outcome $x_{* *}$ exists with $x_{*} \succ x_{* *}$ such that RDU holds for $\succcurlyeq$ on $\mathcal{L}_{\left\{y \in X \mid y \succ x_{* *}\right\}}$. As $(a, b)$ is an interval in $\mathbb{R}$, thus a connected and separable subset, such an outcome exists. Then e-RMC implies that for all $x_{* *}$ ranked below $x_{*}$ and all sets $Z^{\prime \prime}=\left\{z_{1}^{\prime \prime}, \ldots, z_{n^{\prime \prime}}^{\prime \prime}\right\}$ with $x_{* *} \succ z_{1}^{\prime \prime} \succ \cdots \succ z_{n^{\prime \prime}}^{\prime \prime}, n^{\prime \prime} \geq 3$, BMC below $z^{\prime \prime}$ holds and GMC above $z^{\prime \prime}$ holds for all $z^{\prime \prime} \in Z^{\prime \prime}$ when considering the preference $\succcurlyeq$ on $\mathcal{L}_{\left\{x_{* *}\right\} \cup Z^{\prime \prime}}$. Thus, RDU represents $\succcurlyeq$ on $\mathcal{L}_{\left\{x_{* *}\right\}} \cup Z^{\prime \prime}$ for all $x_{* *} \prec x_{*}$ and all sets $Z^{\prime \prime}=\left\{z_{1}^{\prime \prime}, \ldots, z_{n^{\prime \prime}}^{\prime \prime}\right\}$ with $x_{* *} \succ z_{1}^{\prime \prime} \succ \cdots \succ z_{n^{\prime \prime}}^{\prime \prime}, n^{\prime \prime} \geq 3$. Hence, RDU represents $\succcurlyeq$ on $\mathcal{L}_{Z^{\prime \prime}}$ for all finite sets $Z^{\prime \prime}$ with outcomes below $x_{*}$, and therefore, RDU holds for $\succcurlyeq$ on $\mathcal{L}_{\left\{z \in X \mid z \prec x_{*}\right\}}$. This means that $x_{*}$ is the unique point with the property that RDU with the weighting function $w^{+}$holds for $\succcurlyeq$ on $\mathcal{L}_{\left\{y \in X \mid y \succ x_{*}\right\}}$ and RDU with the weighting function $w^{-}$ holds for $\succcurlyeq$ on $\mathcal{L}_{\left\{z \in X \mid z<x_{*}\right\}}$. That is, $r:=x_{*}$ is the unique reference point. 
Next we show that PT with sign-dependence represents $\succcurlyeq$ on $\mathcal{L}$. By e-RMC, one of the following holds:

(a) For all finite sets $Y$ with all outcomes ranked above $r$, BMC below $y$ holds and GMC above $y$ holds for all $y \in Y$ when considering the preference $\succcurlyeq$ on $\mathcal{L}_{Y \cup\{r\}}$. (b) For all finite sets $Z$ with all outcomes ranked below $r$, BMC below $z$ holds and

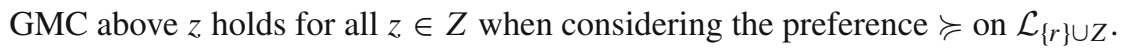

If Subcase III(a) holds, we conclude that $\mathrm{RDU}^{+}$with the weighting function $w^{+}$ and strictly increasing utility $u^{+}$represents the preference $\succcurlyeq$ on $\mathcal{L}_{\{y \in X \mid y \succcurlyeq r\}}$. Further, we know that $\mathrm{RDU}^{-}$with the weighting function $w^{-}$and strictly increasing utility $u^{-}$holds for $\succcurlyeq$ on $\mathcal{L}_{\{z \in X \mid z \prec r\}}$. Also, we know that the general additive representation of $\succcurlyeq$ on $\mathcal{L}$ in Eq. (10) represents the preference on both $\mathcal{L}_{\{y \in X \mid y \succcurlyeq r\}}$ and on $\mathcal{L}_{\{z \in X \mid z \prec r\}}$. This means that, when restricted to the corresponding domain of prospects, $\mathrm{RDU}^{+}$and $\mathrm{RDU}^{-}$agree with the common representation in Eq. (10) if appropriate cardinal transformations of the utility functions are chosen. We determine these cardinal transformations. Consider, for arbitrary finite sets $Y=\left\{y_{1}, \ldots, y_{k-1}\right\}$ of gains and $Z=\left\{z_{k+1}, \ldots, z_{n}\right\}$ of losses for some natural numbers $k<n$, such that $y_{1} \succ \cdots \succ y_{k-1} \succ r \succ z_{k+1} \succ \cdots \succ z_{n}$, the preference over $\mathcal{L}_{Y \cup\{r\} \cup Z \text {. This }}$ preference is represented by

$$
\begin{aligned}
V\left(p_{1}: y_{1}, \ldots, p_{k}: r, \ldots, p_{n}: z_{n}\right)= & \sum_{j=1}^{k-1} V_{j}^{Y \cup\{r\} \cup Z}\left(p_{j}^{d}\right)+V_{k}^{Y \cup\{r\} \cup Z}\left(p_{k}^{d}\right) \\
& +\sum_{j=k+1}^{n} V_{j}^{Y \cup\{r\} \cup Z}\left(p_{j}^{d}\right) .
\end{aligned}
$$

We fix the location parameter for the otherwise jointly cardinal functions as follows:

$$
\begin{aligned}
V_{j}^{Y \cup\{r\} \cup Z}(0) & =0 \text { for } j=1, \ldots, k-1, \\
\text { and } V_{j}^{Y \cup\{r\} \cup Z}(1) & =0 \text { for } j=k, \ldots, n,
\end{aligned}
$$

and we define the functions

$$
\begin{aligned}
\tilde{V}_{j}^{Y \cup\{r\} \cup Z}\left(p_{j}^{c}\right):= & V_{j-1}^{Y \cup\{r\} \cup Z}\left(1-p_{j}^{c}\right) \\
= & V_{j-1}^{Y \cup\{r\} \cup Z}\left(p_{j-1}^{d}\right)
\end{aligned}
$$

for $j=k+1, \ldots, n$. Our additive representation becomes

$$
V\left(p_{1}: y_{1}, \ldots, p_{k}: r, \ldots, p_{n}: z_{n}\right)=\sum_{j=1}^{k-1} V_{j}^{Y \cup\{r\} \cup Z}\left(p_{j}^{d}\right)+\sum_{j=k+1}^{n} \tilde{V}_{j}^{Y \cup\{r\} \cup Z}\left(p_{j}^{c}\right) .
$$

The restriction of the representation in Eq. (11) to $\mathcal{L}_{Y \cup\{r\}}$ and to $\mathcal{L}_{Z}$ also represents the preference over the corresponding set of prospects. The former representation is 
obtained when the probabilities of all losses are equal to 0 , while the latter is obtained when the probabilities of all gains and the reference point are 0 . Thus, for probabilities $p_{1}, \ldots, p_{k}$ that sum to 1 , we have

$$
V\left(p_{1}: y_{1}, \ldots, p_{k}: r\right)=\sum_{j=1}^{k-1} V_{j}^{Y \cup\{r\} \cup Z}\left(p_{j}^{d}\right)
$$

which represents the preference on $\mathcal{L}_{Y \cup\{r\}}$ and, because $\mathrm{RDU}^{+}$also holds on $\mathcal{L}_{Y \cup\{r\}}$, unique numbers $A^{+}>0$ and $B^{+}$exist such that

$$
V\left(p_{1}: y_{1}, \ldots, p_{k}: r\right)=\sum_{j=1}^{k-1} w^{+}\left(p_{j}^{d}\right) A^{+}\left[u^{+}\left(y_{j}\right)-u^{+}\left(y_{j+1}\right)\right]+A^{+} u^{+}(r)+B^{+} .
$$

Therefore,

$$
\begin{aligned}
A^{+} u^{+}(r)+B^{+} & =0 \\
\text { and } V_{j}^{Y \cup\{r\} \cup Z}\left(p_{j}^{d}\right) & =w^{+}\left(p_{j}^{d}\right) A^{+}\left[u^{+}\left(y_{j}\right)-u^{+}\left(y_{j+1}\right)\right]
\end{aligned}
$$

for $j=1, \ldots, k-1$. We define $u(y)=A^{+} u^{+}(y)+B^{+}$for outcomes $y \succcurlyeq r$.

Further, for probabilities $p_{k+1}, \ldots, p_{n}$ that sum to 1

$$
V\left(p_{k+1}: z_{k+1}, \ldots, p_{n}: z_{n}\right)=\sum_{j=k+1}^{n} \tilde{V}_{j}^{Y \cup\{r\} \cup Z}\left(p_{j}^{c}\right)
$$

represents the preference on $\mathcal{L}_{Z}$ and, because $\mathrm{RDU}^{-}$also holds on $\mathcal{L}_{Z}$, unique numbers $A^{-}>0$ and $B^{-}$exist such that

$$
\begin{aligned}
V\left(p_{k+1}: z_{k+1}, \ldots, p_{n}: z_{n}\right)=B^{-} & +A^{-} u^{-}\left(z_{k+1}\right) \\
& +\sum_{j=k+1}^{n} \tilde{w}^{-}\left(p_{j}^{c}\right) A^{-}\left[u^{-}\left(z_{j}\right)-u^{-}\left(z_{j-1}\right)\right] .
\end{aligned}
$$

We define $u(z)=A^{-} u^{-}(z)+B^{-}$for outcomes $z \prec r$. Substitution into the representation in Eq. (11) gives

$$
\begin{aligned}
V\left(p_{1}: y_{1}, \ldots, p_{k}: r, \ldots, p_{n}: z_{n}\right)= & \sum_{j=1}^{k-1} V_{j}^{Y \cup\{r\} \cup Z}\left(p_{j}^{d}\right)+\sum_{j=k+1}^{n} \tilde{V}_{j}^{Y \cup\{r\} \cup Z}\left(p_{j}^{c}\right) \\
= & \sum_{j=1}^{k-1} w^{+}\left(p_{j}^{d}\right)\left[u\left(y_{j}\right)-u\left(y_{j+1}\right)\right]+ \\
& u\left(z_{k+1}\right)+\sum_{j=k+1}^{n} \tilde{w}^{-}\left(p_{j}^{c}\right)\left[u\left(z_{j}\right)-u\left(z_{j-1}\right)\right],
\end{aligned}
$$


which is a PT-representation with the uniquely determined weighting functions. We have fixed the location parameters for each of the additive functions $V_{j}^{Y \cup\{r\} \cup Z}$, which makes them joint ratio scales. For the utility function $u$ this means that it must be a ratio scale.

As $Y$ and $Z$ were arbitrary sets of gains and losses, respectively, it follows that the general additive representation in Eq. (10) is indeed a PT-representation. Hence, PT represents $\succcurlyeq$ on $\mathcal{L}$. This completes the derivation for Subcase III(a). In particular, as $u(r)=0$, it follows that $\mathrm{RDU}^{-}$represents the preference on the larger set $\mathcal{L}_{\{z \in X \mid z \preccurlyeq r\}}$ rather than just on $\mathcal{L}_{\{z \in X \mid z<r\}}$.

If Subcase III(b) holds, we conclude that $\mathrm{RDU}^{+}$with the weighting function $w^{+}$and the strictly increasing utility $u^{+}$represents the preference $\succcurlyeq$ on $\mathcal{L}_{\{y \in X \mid y \succ r\}}$. Further, we know that $\mathrm{RDU}^{-}$with weighting function $w^{-}$and strictly increasing utility $u^{-}$ holds for $\succcurlyeq$ on $\mathcal{L}_{\{z \in X \mid z \preccurlyeq r\}}$. Also, we know that the general additive representation of $\succcurlyeq$ on $\mathcal{L}$ in Eq. (10) represents the preference on both $\mathcal{L}_{\{y \in X \mid y \succ r\}}$ and $\mathcal{L}_{\{z \in X \mid z \preccurlyeq r\}}$. As in Subcase III(a), we look for specific cardinal transformations for the utility functions in $\mathrm{RDU}^{+}$and $\mathrm{RDU}^{-}$such that they agree with the common representation in Eq. (10). The analysis is similar to Subcase III(a) except that $\mathrm{RDU}^{-}$holds on $\mathcal{L}_{\{r\} \cup Z}$, which means that when fixing the location parameters for the functions in the additive representation we obtain

$$
A^{-} u^{-}(r)+B^{-}=0=V_{k}^{Y \cup\{r\} \cup Z}(1) .
$$

We conclude that also in Subcase III(b) PT represents $\succcurlyeq$ on $\mathcal{L}$. Similar to Subcase III(a) it follows that $\mathrm{RDU}^{+}$represents the preference on the larger set $\mathcal{L}_{\{y \in X \mid y \succcurlyeq r\}}$ (instead of just $\mathcal{L}_{\{y \in X \mid y \succ r\}}$ ). This completes the proof for Case III.

CASE IV: In this case we consider the remaining possibility, that for an outcome $x \in X$ and a set $Y=\left\{y_{1}, \ldots, y_{n}\right\}$ with $y_{1} \succ \cdots \succ y_{n} \succ x, n \geq 3$, for the preference $\succcurlyeq$ on $\mathcal{L}_{Y \cup\{x\}}$ BMC below $y$ does not hold for an outcome $y \in Y$ or GMC above $y^{\prime}$ does not hold for an outcome $y^{\prime} \in Y$. In particular, this means that RDU does not hold for the preference $\succcurlyeq$ on $\mathcal{L}_{Y \cup\{x\}}$. Exploiting e-RMC and using similar arguments to those presented in Case III, we can identify the reference point and subsequently show that PT represents the preference on $\mathcal{L}$. This completes Case IV.

As Cases I-IV exhaust all possibilities, statement (i) of Theorem 3 has been derived. This concludes the proof of Theorem 3 .

Proof of Theorem 4 That statement (i) implies statement (ii) is standard; it follows from the properties of the PT-functional. We prove that statement (ii) implies statement (i). That the preference is represented by a general additive representation follows by similar arguments as in the proof of Theorem 3. Cases I and II are completely analogous. In particular, they follow from the results in Zank (2010).

For the analog to Cases III and IV, the arguments are similar to those in the proof of Theorem 3, except that a reference point within $X$ may not exist. From e-RMC it follows that the set of outcomes contains two sets $Y$ and $Z$ with $Y \cup Z=X$ such that the preference on $\mathcal{L}_{Y}$ is represented by $\mathrm{RDU}^{+}$and the preference on $\mathcal{L}_{Z}$ is represented by RDU $^{-}$. If the sets $Y$ and $Z$ are not disjoint they contain a unique reference point 
$r$. Then, PT can be derived using arguments similar to those in Case III in the proof of Theorem 3.

If $Y$ and $Z$ are disjoint, then $Y$ contains only gains and $Z$ contains only losses; no reference point within $X$ exists, even though preferences are sign-dependent (i.e., $w^{+} \neq w^{-}$). Similar to Case III in the proof of Theorem 3, we normalize the additive functions for gains and for losses and choose the unique cardinal transformations of $\mathrm{RDU}^{+}$and $\mathrm{RDU}^{-}$to obtain PT. Hence, we obtain statement (i) and the corresponding uniqueness results. This concludes the proof of Theorem 4.

\section{References}

Abdellaoui, M.: Parameter-free elicitation of utility and probability weighting functions. Manag. Sci. 46, 1497-1512 (2000)

Abdellaoui, M.: A genuine rank-dependent generalization of the von Neumann-Morgenstern expected utility theorem. Econometrica 70, 717-736 (2002)

Abdellaoui, M., Baillon, A., Placido, L., Wakker, P.P.: The rich domain of uncertainty: source functions and their experimental implementation. Am. Econ. Rev. 101, 695-723 (2011)

Abdellaoui, M., l'Haridon, O., Zank, H.: Separating curvature and elevation: a parametric probability weighting function. J. Risk Uncertain. 41, 39-65 (2010)

Abdellaoui, M., Vossmann, F., Weber, M.: Choice-based elicitation and decomposition of decision weights for gains and losses under uncertainty. Manag. Sci. 51, 1384-1399 (2005)

Abdellaoui, M., Wakker, P.P.: The likelihood method for decision under uncertainty. Theor. Decis. 58, 3-76 (2005)

Allais, M.: Le Comportement de l'Homme Rationnel devant le Risque: Critique des Postulats et Axiomes de l'Ecole Américaine. Econometrica 21, 503-546 (1953)

al-Nowaihi, A., Dhami, S.: Composite Prospect Theory. Working Paper No. 10/11, Department of Economics, University of Leicester (2010)

Andreoni J., Sprenger, C.: Certain and Uncertain Utility: The Allais Paradox and Five Decision Theory Phenomena. Working Paper UCSD (2010)

Andreoni, J., Sprenger, C.: Risk preferences are not time preferences. Am. Econ. Rev. 102, 3357-3376 (2012)

Apesteguia, J., Ballester, M.A.: A theory of reference-dependent behavior. Econ. Theor. 40, 427-455 (2009)

Baillon, A., Driesen, B., Wakker, P.P.: Relative concave utility for risk and ambiguity. Games Econ. Behav. 75, 481-489 (2012)

Barberis, N.: Thirty years of prospect theory in economics: a review and assessment. J. Econ. Perspect. 27, 173-195 (2013)

Barberis, N., Huang, M., Santos, T.: Prospect theory and asset prices. Quart. J. Econ. 116, 1-53 (2001)

Bell, D.E.: Disappointment in decision making under uncertainty. Oper. Res. 33, 1-27 (1985)

Birnbaum, M.H., Stegner, S.E.: Measuring the importance of cues in judgment for individuals: subjective theories of IQ as a function of heredity and environment. J. Exp. Soc. Psychol. 17, 159-182 (1981)

Blavatskyy, P.: Loss aversion. Econ. Theory 46, 127-148 (2011). https://doi.org/10.1007/s00199-0090504-7

Bleichrodt, H.: Reference-dependent utility with shifting reference points and incomplete preferences. J. Math. Psychol. 51, 266-276 (2007)

Bleichrodt, H., Pinto, J.L.: A parameter-free elicitation of the probability weighting function in medical decision analysis. Manag. Sci. 46, 1485-1496 (2000)

Bossert, W., Sprumont, Y.: Non-deteriorating choice. Economica 76, 337-363 (2009)

Chateauneuf, A.: Comonotonicity axioms and rank-dependent expected utility for arbitrary consequences. J. Math. Econ. 32, 21-45 (1999)

Chateauneuf, A., Eichberger, J., Grant, S.: Choice under Uncertainty with the best and worst in mind: NEO-additive capacities. J. Econ. Theory 137, 538-567 (2007)

Chateauneuf, A., Wakker, P.P.: An axiomatization of cumulative prospect theory for decision under risk. J. Risk Uncertain. 18, 137-145 (1999)

Chew, S.H., Wakker, P.P.: The comonotonic sure-thing principle. J. Risk Uncertain. 12, 5-27 (1996) 
Cohen, M.: Security level, potential level, expected utility: a three-criteria decision model under risk. Theor. Decis. 33, 101-134 (1992)

Cubitt, R.P., Starmer, C., Sugden, R.: On the validity of the random lottery incentive system. Exp. Econ. 1, 115-131 (1998)

Debreu, G.: Representation of a Preference Ordering by a Numerical Function. In: Thrall, R.M., Coombs, C.H., Davis, R.L. (eds.) Decision Processes, pp. 159-165. Wiley, New York (1954)

Delquié, P., Cillo, A.: Disappointment without prior expectation: a unifying perspective on decision under risk. J. Risk Uncertain. 33, 197-215 (2006)

Diecidue, E., Schmidt, U., Zank, H.: Parametric weighting functions. J. Econ. Theory 144, 1102-1118 (2009)

Edwards, W.: Probability-preferences in gambling. Am. J. Psychol. 66, 349-364 (1953)

Edwards, W.: Probability-preferences among bets with differing expected values. Am. J. Psychol. 67, 56-67 (1954)

Ellsberg, D.: Risk, ambiguity and the savage axioms. Quart. J. Econ. 75, 643-669 (1961)

Etner, J., Jeleva, M.: Underestimation of probabilities modifications: characterization and economic implications. Econ. Theory 56, 291-307 (2014). https://doi.org/10.1007/s00199-013-0781-z

Fudenberg, D.: Advancing beyond advances in behavioral economics. J. Econ. Lit. 44, 694-711 (2006)

Gul, F.: A theory of disappointment aversion. Econometrica 59, 667-686 (1991)

Herstein, I.N., Milnor, J.: An axiomatic approach to measurable utility. Econometrica 21, 291-297 (1953)

Holt, C.A., Laury, S.K.: Risk aversion and incentive effects. Am. Econ. Rev. 92, 1644-1655 (2002)

Kahneman, D., Tversky, A.: Prospect theory: an analysis of decision under risk. Econometrica 47, 263-291 (1979)

Kahneman, D., Tversky, A.: Choices, Values, and Frames. Cambridge University Press, New York (2000)

Köbberling, V., Wakker, P.P.: Preference foundations for nonexpected utility: a generalized and simplified technique. Math. Oper. Res. 28, 395-423 (2003)

Köbberling, V., Wakker, P.P.: An index of loss aversion. J. Econ. Theory 122, 119-131 (2005)

Kőszegi, B.: Utility from anticipation and personal equilibrium. Econ. Theory 44, 415-444 (2010). https:// doi.org/10.1007/s00199-009-0465-X

Kőszegi, B., Rabin, M.: A model of reference-dependent preferences. Quart. J. Econ. 121, 1133-1165 (2006)

Kőszegi, B., Rabin, M.: Reference-dependent risk attitudes. Am. Econ. Rev. 97, 1047-1073 (2007)

Kothiyal, A., Spinu, V., Wakker, P.P.: Prospect Theory for continuous distributions: a preference foundation. J. Risk Uncertain. 42, 195-210 (2011)

Kunreuther, H., Ginsberg, R., Miller, L., Sagi, P., Slovic, P., Borkan, B., Katz, N.: Disaster Insurance Protection: Public Policy Lessons. Wiley Interscience, New York (1978)

Machina, M.J.: Dynamic consistency and non-expected utility models of choice under uncertainty. J. Econ. Lit. 27, 1622-1688 (1989)

Munro, A., Sugden, R.: On the theory of reference-dependent preferences. J. Econ. Behav. Organ. 50, 407-428 (2003)

Nakamura, Y.: Rank dependent utility for arbitrary consequence spaces. Math. Soc. Sci. 29, 103-129 (1995)

Neilson, W.S.: Comparative risk sensitivity with reference-dependent preferences. J. Risk Uncertain. 24, 131-142 (2002)

Neilson, W.S.: Axiomatic reference-dependence in behavior toward others and toward risk. Econ. Theory 28, 681-692 (2006). https://doi.org/10.1007/s00199-005-0643-4

Ok, E.A., Ortoleva, P., Riella, G.: Revealed (P)reference theory. Am. Econ. Rev. 105, 299-321 (2015)

Palm, R.I.: Earthquake Insurance: A Longitudinal Study of California Homeowners. Westview Press, Boulder (1995)

Pesendorfer, W.: Behavioral economics comes of age: a review essay on advances in behavioral economics. J. Econ. Lit. 44, 712-721 (2006)

Prelec, D.: The probability weighting function. Econometrica 66, 497-527 (1998)

Preston, M.G., Baratta, P.: An experimental study of the auction value of an uncertain outcome. Am. J. Psychol. 61, 183-193 (1948)

Quiggin, J.: Risk perception and risk aversion among Australian farmers. Aust. J. Agric. Econ. 25, 160-169 (1981)

Quiggin, J.: A theory of anticipated utility. J. Econ. Behav. Organ. 3, 323-343 (1982)

Sagi, J.S.: Anchored preference relations. J. Econ. Theory 130, 283-295 (2006)

Savage, L.J.: The Foundations of Statistics. Wiley, New York (1954) 
Schmeidler, D.: Subjective probability and expected utility without additivity. Econometrica 57, 571-587 (1989)

Schmidt, U., Starmer, C., Sugden, R.: Third-generation Prospect Theory. J. Risk Uncertain. 36, 203-223 (2008)

Schmidt, U., Zank, H.: A genuine foundation for prospect theory. J. Risk Uncertain. 45, 97-113 (2012)

Segal, U.: Some remarks on Quiggin's anticipated utility. J. Econ. Behav. Organ. 8, 145-154 (1987)

Shalev, J.: Loss aversion equilibrium. Int. J. Game Theory 29, 269-287 (2000)

Shalev, J.: Loss aversion and bargaining. Theor. Decis. 52, 201-232 (2002)

Shleifer, A.: Psychologists at the Gate: A Review of Daniel Kahneman's Thinking, Fast and Slow. Book review of: Kahneman, D. (2011) “Thinking: Fast and Slow," Penguin Books. London. J. Econ. Lit. 50, 1080-1091 (2012)

Starmer, C.: Developments in non-expected utility theory: the hunt for a descriptive theory of choice under risk. J. Econ. Lit. 38, 332-382 (2000)

Sugden, R.: Reference-dependent subjective expected utility. J. Econ. Theory 111, 172-191 (2003)

Thaler, R.H., Johnson, E.J.: Gambling with the house money and trying to break even: the effects of prior outcomes on risky choice. Manag. Sci. 36, 643-660 (1990)

Tversky, A., Kahneman, D.: Advances in prospect theory: cumulative representation of uncertainty. J. Risk Uncertain. 5, 297-323 (1992)

van de Kuilen, G., Wakker, P.P.: The midweight method to measure attitudes toward risk and ambiguity. Manag. Sci. 57, 582-598 (2011)

Viscusi, K.W., Magat, W.A., Huber, J.: An investigation of the rationality of consumer valuations of multiple health risks. Rand J. Econ. 18, 465-479 (1987)

von Neumann, J.., Morgenstern, O.: (1944, 1947, 1953) Theory of Games and Economic Behavior. Princeton University Press, Princeton

Wakker, P.P.: Additive representations on rank-ordered sets: II. The topological approach. J. Math. Econ. 22, 1-26 (1993)

Wakker, P.P.: Separating marginal utility and probabilistic risk aversion. Theor. Decis. 36, 1-44 (1994)

Wakker, P.P.: Testing and characterizing properties of nonadditive measures through violations of the surething principle. Econometrica 69, 1039-1059 (2001)

Wakker, P.P.: Prospect Theory For Risk and Ambiguity. Cambridge University Press, Cambridge (2010)

Wakker, P.P., Deneffe, D.: Eliciting von Neumann-Morgenstern utilities when probabilities are distorted or unknown. Manag. Sci. 42, 1131-1150 (1996)

Wakker, P.P., Tversky, A.: An axiomatization of cumulative prospect theory. J. Risk Uncertain. 7, 147-176 (1993)

Webb, C.S., Zank, H.: Accounting for optimism and pessimism in expected utility. J. Math. Econ. 47, 706-717 (2011)

Webb, C.S.: Piecewise additivity for non-expected utility. Econ. Theory 60, 371-392 (2015). https://doi. org/10.1007/s00199-015-0871-1

Webb, C.S.: Piecewise linear rank-dependent utility. Theor. Decis. 82, 403-414 (2017)

Wu, G., Gonzalez, R.: Curvature of the probability weighting function. Manag. Sci. 42, 1676-1690 (1996)

Zank, H.: Consistent probability attitudes. Econ. Theory 44, 167-185 (2010). https://doi.org/10.1007/ s00199-009-0484-7 